\title{
COUP-TFII regulates satellite cell function and muscular dystrophy
}

\author{
Xin Xie, ${ }^{1}$ Sophia Y. Tsai,, ${ }^{1,2,3}$ and Ming-Jer Tsai ${ }^{1,2,3}$ \\ ${ }^{1}$ Department of Molecular and Cellular Biology, ${ }^{2}$ Program in Developmental Biology, ${ }^{3}$ Department of Medicine, Baylor College of Medicine, Houston, Texas, USA.
}

\begin{abstract}
Duchenne muscular dystrophy (DMD) is a severe and progressive muscle-wasting disease caused by mutations in the dystrophin gene. Although dystrophin deficiency in myofiber triggers the disease's pathological changes, the degree of satellite cell (SC) dysfunction defines disease progression. Here, we have identified chicken ovalbumin upstream promotertranscription factor II (COUP-TFII) hyperactivity as a contributing factor underlying muscular dystrophy in a dystrophindeficient murine model of DMD. Ectopic expression of COUP-TFII in murine SCs led to Duchenne-like dystrophy in the muscles of control animals and exacerbated degenerative myopathies in dystrophin-deficient mice. COUP-TFII-overexpressing mice exhibited regenerative failure that was attributed to deficient SC proliferation and myoblast fusion. Mechanistically, we determined that COUP-TFII coordinated a regenerative program through combined regulation of multiple promyogenic factors. Furthermore, inhibition of COUP-TFII preserved SC function and counteracted the muscle weakness associated with Duchenne-like dystrophy in the murine model, suggesting that targeting COUP-TFII is a potential treatment for DMD. Together, our findings reveal a regulatory role of COUP-TFII in the development of muscular dystrophy and open up a potential therapeutic opportunity for managing disease progression in patients with DMD.
\end{abstract}

\section{Introduction}

Skeletal muscle is the most abundant tissue in the body and is important for critical functions such as movement, posture maintenance, and whole-body metabolism. It has been known for more than a century that skeletal muscle possesses an extraordinary capacity for self-repair, a process that is absolutely dependent on a small population of stem/progenitor cells, termed satellite cells (SCs) (1-3). In resting muscles, SCs remain quiescent. Upon exposure to environmental insults, SCs exit their dormant state, quickly expand. Most of these SCs differentiate and either fuse with existing fibers or give rise to new myofibers, thereby fully restoring damaged tissue $(2,3)$. Any abnormalities in SC specification, proliferation, differentiation, or self-renewal will lead to muscle atrophy and can aggravate chronic muscle diseases such as Duchenne muscular dystrophy (DMD).

$\mathrm{DMD}$, the most common lethal genetic disorder in children, affects approximately 1 in 3,500 live-born males worldwide (4). Loss of dystrophin in patients causes muscle membrane fragility, which results in repetitive myofiber degeneration and regeneration and the replacement of muscles by fibrofatty tissues and ultimately leads to progressive paralysis and premature death (4). The $m d x$ mouse, which bears the same genetic defect as that found in patients with DMD, reproduces the degenerative changes and is the most frequently used mouse model in DMD research $(5,6)$. Despite recent advances in DMD treatment, current therapies do not cure the disease (4), highlighting the need for a new strategy and a better understanding of the molecular events underlying DMD etiology.

Conflict of interest: The authors have declared that no conflict of interest exists Submitted: March 3, 2016; Accepted: August 2, 2016.

Reference information: / Clin Invest. 2016;126(10):3929-3941. doi:10.1172/JCI87414.
Chicken ovalbumin upstream promoter-transcription factor II (COUP-TFII), also known as NR2F2, is a member of the nuclear orphan receptor superfamily. COUP-TFII is broadly detected in stem/progenitor cells in multiple tissues and has a profound impact on adult stem cell biology (7-9). COUP$\mathrm{TFII}^{-/-}$murine embryos suffer early embryonic lethality owing to defective angiogenesis and cardiac development (10). Initial evidence of a role for COUP-TFII in myogenesis emerged from in vitro cell-based studies (11-13); however, little is known about its in vivo function in muscle development and diseases. In an effort to search for new players that contribute to muscular dystrophy, we queried multiple microarray data sets and found that COUP-TFII transcripts were enriched in patients with DMD compared with those in healthy individuals $(14,15)$. Considering that COUP-TFII protein is primarily detected in SCs in resting muscles (Figure 1, A and B), we generated mouse models expressing COUP-TFII protein specifically in the stem cell compartment. Early activation of COUP-TFII induces dystrophy-like defects in normal muscles that progressively worsen with aging. Consistently, SC-specific COUP-TFII activation exacerbates muscle wasting in the $m d x$ mutant and hinders regenerative responses in WT mice suffering transient injury. The inefficient tissue repair arises from deficient myoblast expansion and fusion and correlates with the dysregulation of cyclin D1 (Ccnd1), myogenic factor 5 (Myf5), caveolin 3 (Cav3), and chemokine (C-X-C motif) receptor 4 (Cxcr4) genes. Furthermore, mice with concurrent loss of COUP-TFII and dystrophin show improved musculature and are protected from progressive myopathies. Together, our findings demonstrate the previously unrecognized effects of COUP-TFII on modulating SC behavior and highlight it as a potential candidate for therapeutic intervention of degenerative muscle diseases. 
A

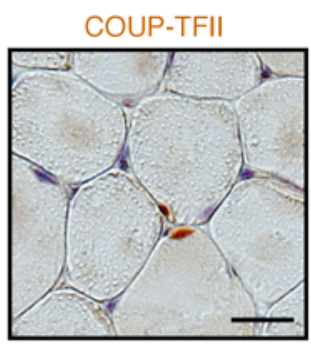

D
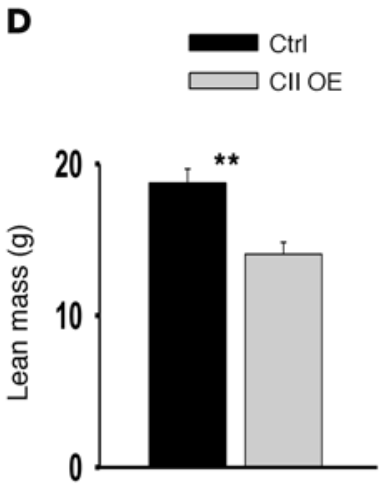

G

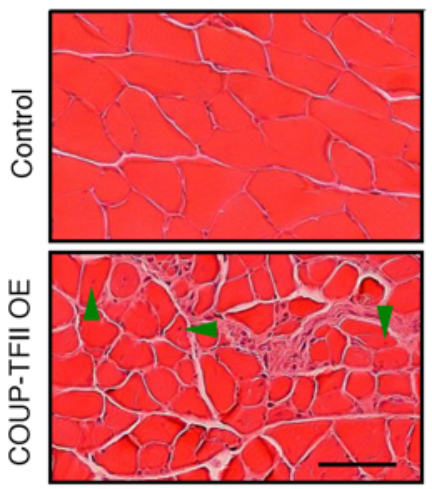

B
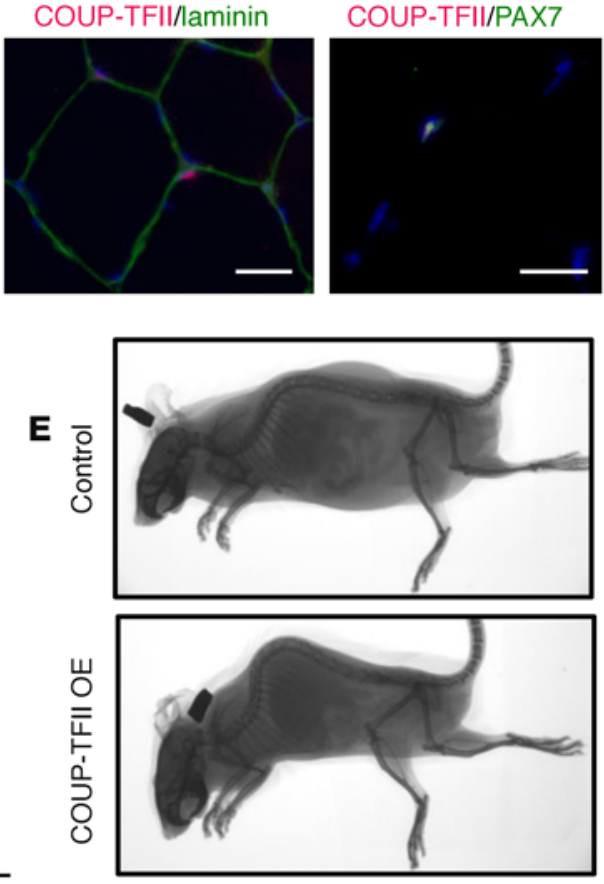

H

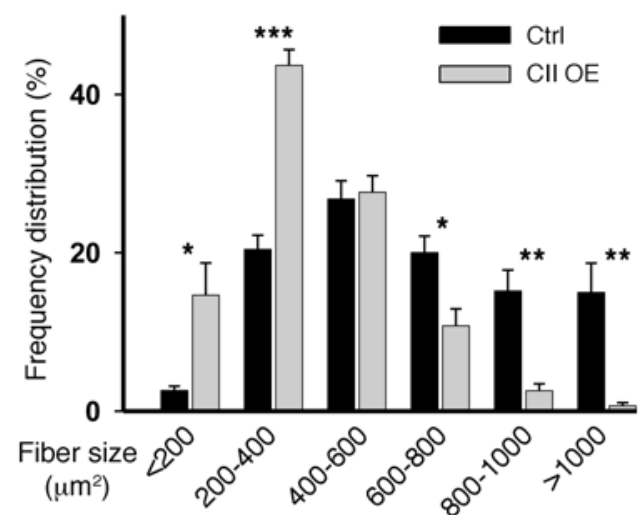

C
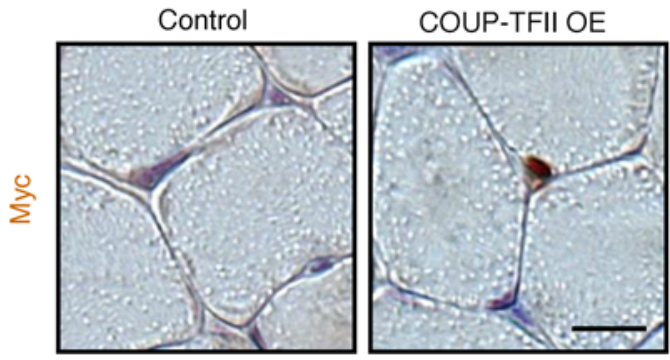

$\mathbf{F}$
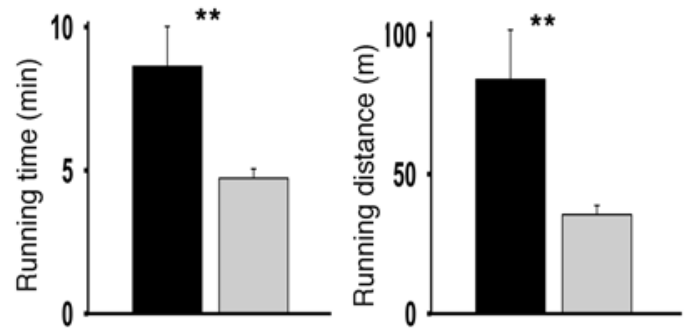

I

$\mathrm{eMHC}$

Trichrome

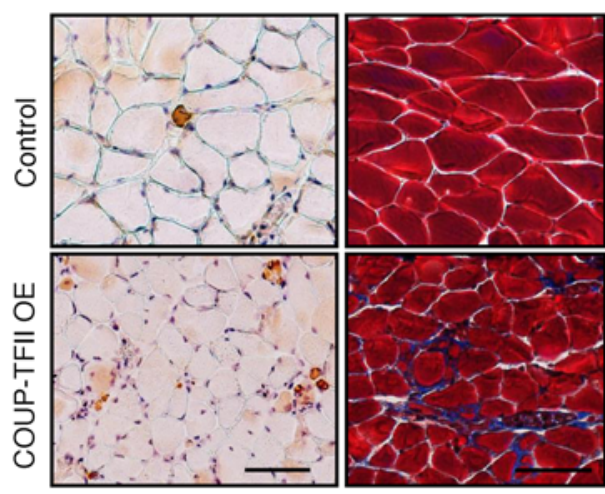

Figure 1. Transgenic expression of COUP-TFII induces dystrophic symptoms upon aging. (A and B) WT TA muscles were stained for COUP-TFII, PAX7, and laminin to illustrate the expression of COUP-TFII. (C) Myc staining indicates COUP-TFII-expressing SCs in TA muscles 1 week after tamoxifen injection. Image is representative of 5 independent experiments. (D) Lean body mass in control (CTRL, $n=5$ ) and COUP-TFII OE (CII OE, $n=8)$ mice. (E) Representative images highlight spinal curvature in COUP-TFII-transgenic animals. (F) Time and distance to fatigue with forced treadmill running in the control $(n=7)$ and COUP-TFII OE $(n=6)$ animals. (G) Representative images of H\&E-stained soleus muscles and (H) quantification of muscle fiber caliber in control $(n=6)$ and COUP-TFII OE $(n=5)$ mice. Arrowheads in $\mathbf{G}$ indicate the central-nucleated fibers. (I) eMHC- and trichrome-stained soleus muscles. Fibrosis was analyzed by trichrome staining (right). Scale bars: $20 \mu \mathrm{m}$ (A and $\mathbf{B}), 10 \mu \mathrm{m}$ (C), $50 \mu \mathrm{m}$ (G and I). ${ }^{*} P<0.05,{ }^{* *} P<0.01$, and ${ }^{* * *} P<0.001$, by Student's $t$ test. Data represent the mean $\pm \mathrm{SEM}$.

\section{Results}

COUP-TFII-overexpressing mice develop Duchenne-like dystrophy upon aging. In patients with $\mathrm{DMD}$, dystrophin is lost in muscles in the fetal stage, while the deterioration of muscle function is progressive, suggesting that there are critical secondary factors that compound the dystrophin deficit. To identify novel molecules acting downstream of dystrophin loss, we interrogated public databases for differentially regulated genes between patients with muscular disorders and age-matched healthy individuals. Among the variably expressed genes, COUP-TFII mRNA was upregulated in patients with DMD (Supplemental Figure 1A; supplemental material available online with this article; doi:10.1172/ JCI87414DS1) $(14,15)$. Similarly, an elevated level of COUP-TFII was found in the diaphragm muscle of $m d x$ mice (Supplemental Figure 1B). Interestingly, while muscles from dystrophic patients have higher levels of COUP-TFII, treatments that ameliorate clinical phenotypes reduce COUP-TFII transcription (16). The potential link between COUP-TFII and muscular dystrophy is further substantiated by the fact that many genes affiliated with genetic or acquired myopathies are differentially expressed in 
A

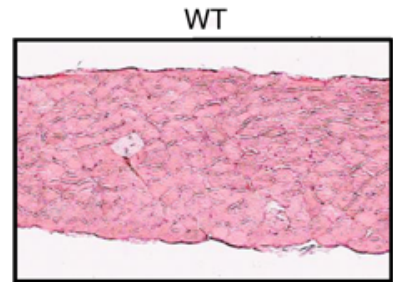

C

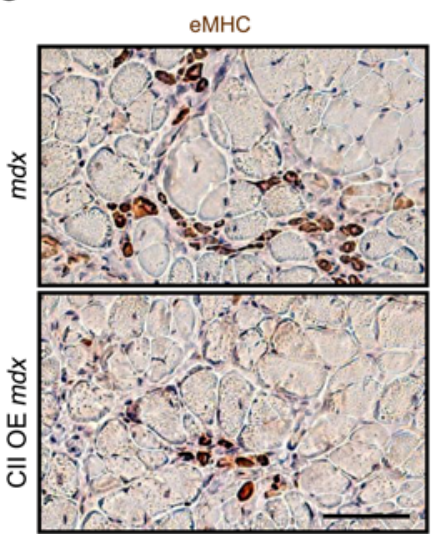

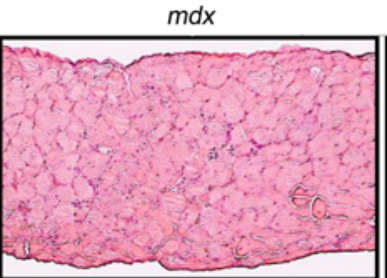

D

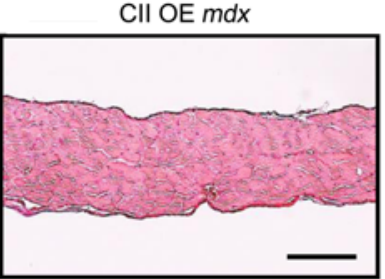

E

B

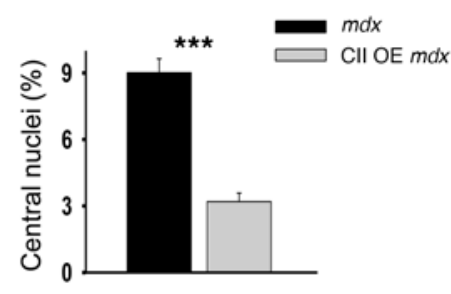

$\mathbf{F}$
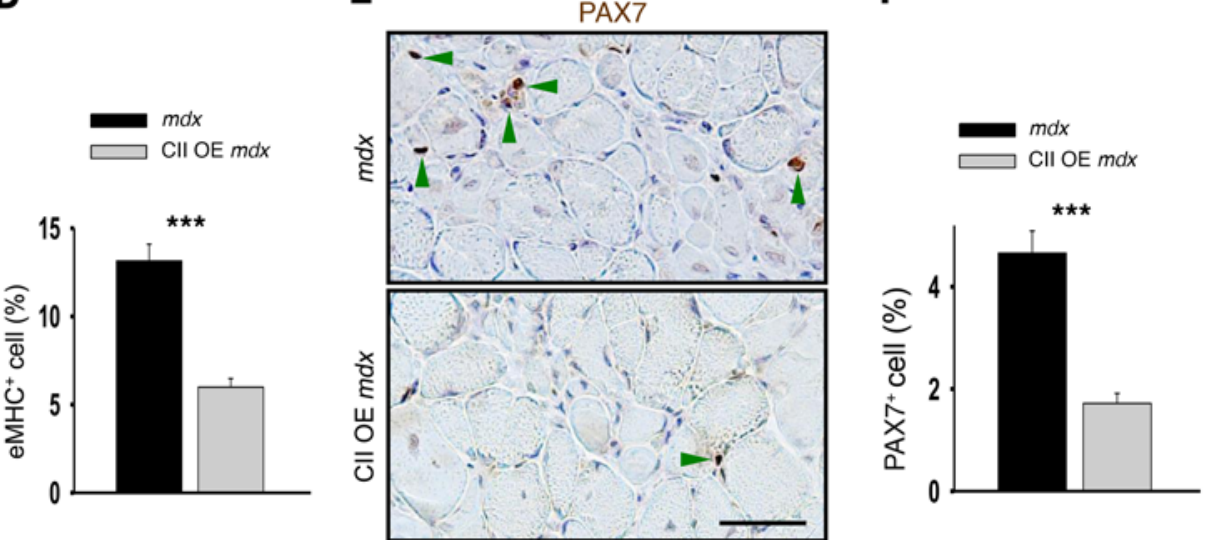

G

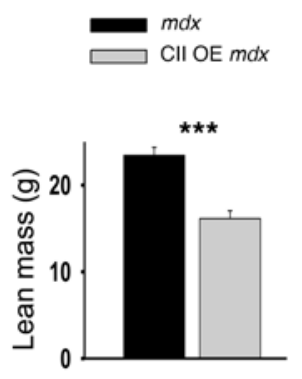

H

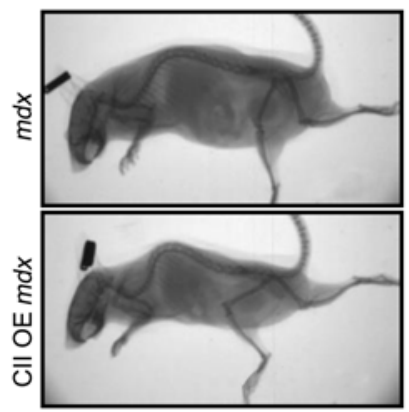

I

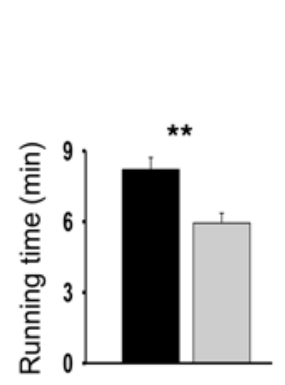

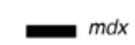

$\square$ CII OE $m d x$

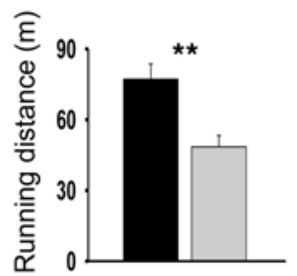

J
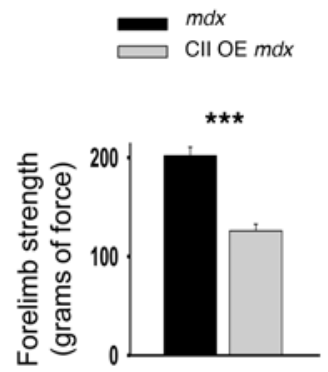

Figure 2. Analyses of the exacerbated dystrophic phenotypes in COUP-TFII OE $\boldsymbol{m} \boldsymbol{d x}$ mice. (A) Representative H\&E-stained diaphragm muscles from the indicated genotypes at 4 months of age. (B) Histogram indicates the percentage of central nuclei in the total nuclei in diaphragm muscles from $m d x(n=6$ ) mice and COUP-TFII OE $m d x(n=8)$ mice. (C and $\mathbf{D})$ Muscles were stained for eMHC, and eMHC fibers were measured as a fraction of total nuclei in $m d x$ $(n=9)$ and COUP-TFII OE $m d x(n=7)$ strains. (E and F) Representative image and quantification of PAX7-expressing SCs in diaphragm sections in $m d x(n=8)$ and COUP-TFII OE $m d x(n=10)$ mouse strains. Arrowheads indicate the PAX7+ SCs. (G) Average mean value of lean body mass in $m d x(n=7)$ and COUP-TFII $\mathrm{OE} m d x(n=6)$ mouse strains. (H) Representative $x$-ray image indicates severe kyphosis in dystrophic mice carrying the COUP-TFII minigene at 8 months of age. (I and J) Treadmill and grip tests for 6-month-old $m d x(n=12)$ and COUP-TFII OE $m d x(n=10)$ animals. Scale bars: $100 \mu \mathrm{m}$ (A), $50 \mu \mathrm{m}$ (C), $25 \mu \mathrm{m}$ (E). ${ }^{* *} P<0.01$ and ${ }^{* *} P<0.001$, by Student's $t$ test. Data represent the mean $\pm \mathrm{SEM}$.

COUP-TFII-dysregulated cells (17-19). In agreement with this, aberrant COUP-TFII activity has been connected to human diseases including congenital diaphragmatic hernia, prostate cancer, and heart failure (18-20). Together, these observations support a hypothesis that COUP-TFII may play a role in muscular dystrophy.

In uninjured muscles, COUP-TFII protein was primarily identified in the characteristic SC position, as COUP-TFII ${ }^{+}$cells resided underneath the surrounding basal lamina and coexpressed the SC marker paired box 7 (PAX7) (Figure 1, A and B). Using COUP-TFII-LacZ-knockin mice (21), we verified the SC-specific location of COUP-TFII, because $\beta$-gal signals were recognized in small, de novo fibers with centrally positioned myonuclei on days 8 and 12 after injury (Supplemental Figure 1C). Therefore, we reasoned that the SC compartment may represent the central site of
COUP-TFII function in muscle development and disease. To test this, we used an inducible $\operatorname{Pax} 7^{\mathrm{CreRRT2} /+}$ mouse strain to achieve ectopic expression of COUP-TFII protein in SCs (COUP-TFII OE) (22). One week after tamoxifen-stimulated recombination, the Myc-tagged COUP-TFII transgene (23) was exclusively found in the SC position in muscle fibers (Figure 1C). More important, the extent of COUP-TFII overexpression in our transgenic animal was comparable to the levels observed in DMD patients and $m d x$ mice (Supplemental Figure 1, A and B). Further analyses using freshly isolated SCs revealed an approximately 6-fold induction of COUPTFII within the stem cell population (Supplemental Figure 1D).

Unexpectedly, at 20 months of age, mice harboring the COUP-TFII transgene developed many features resembling pathological hallmarks of human DMD $(24,25)$. For instance, 
A

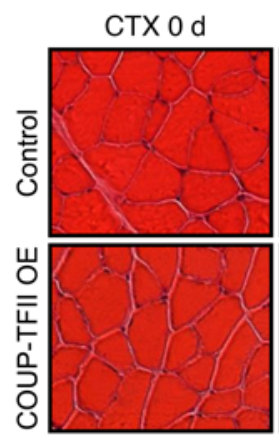

C

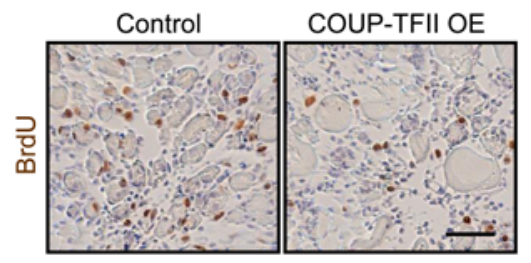

G

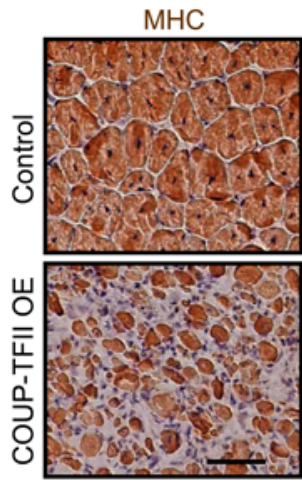

CTX $2 d$

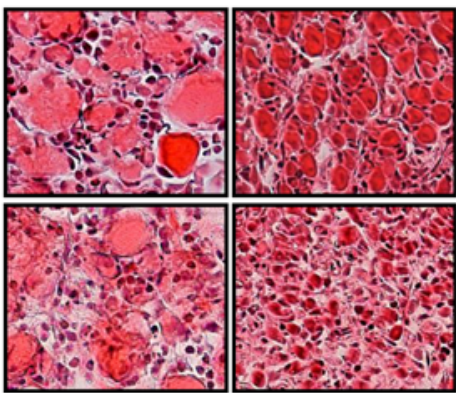

D

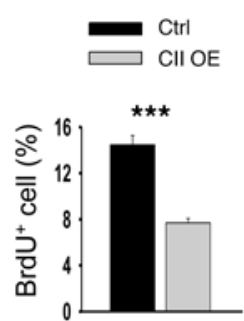

B

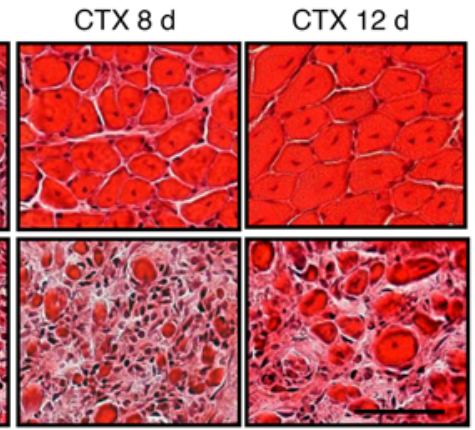

E

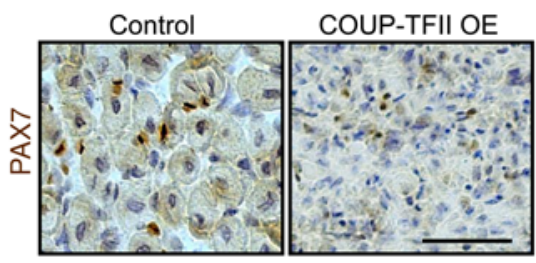

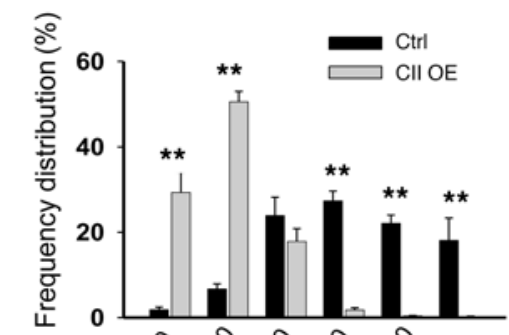

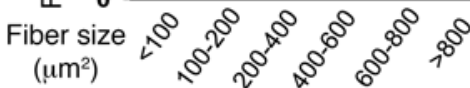

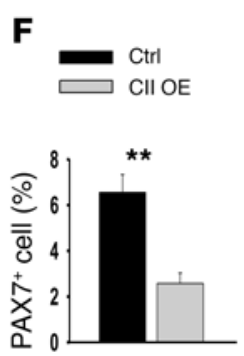

H

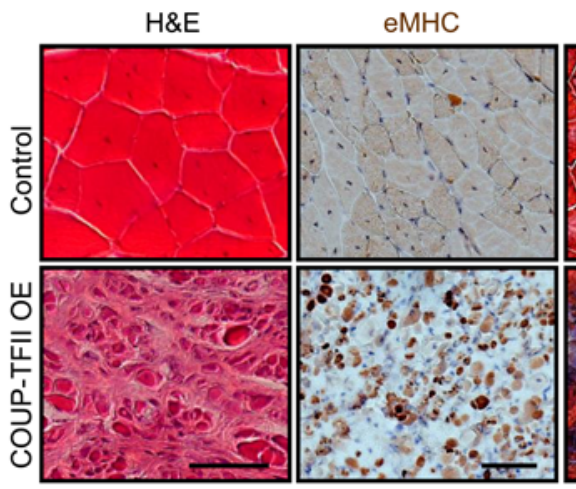

Trichrome

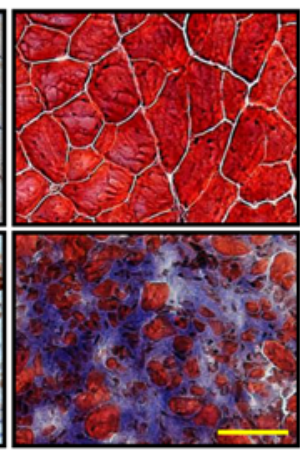

Figure 3. Impaired muscle recovery in COUP-TFII-transgenic mice suffering acute injury. (A) Representative H\&E-stained TA muscles showed the course of regeneration in control and COUP-TFII OE mice at different time points after injury. Images are representative of 4 animals for each genotype at different time points. (B) Histograms represent the frequency distribution across TA muscles according to the cross-sectional area at post-damage day 12 ( $n=6$ ). (C and D) Two days after injury, TA muscle were pulsed with BrdU for 2 hours prior to tissue harvesting and were stained with BrdU Ab. Percentage of BrdU+ cells over total nuclei in control $(n=5)$ and COUP-TFII OE $(n=6)$ mice. (E and F) Representative images of PAX7 immunostaining and quantitative measurement of PAX7+ cells 5 days after muscle damage $(n=6)$. (G) Immunostaining for MHC, eMHC, and Myc 12 days after chemical injection. (H) TA muscles were examined 2 months after CTX-induced injury. Data are representative of 3 independent experiments. Scale bars: $50 \mu \mathrm{m}$. ${ }^{* *} P<0.01$ and ${ }^{* * *} P<0.001$, by 2-tailed Student's $t$ test. Data represent the mean \pm SEM.

COUP-TFII-transgenic mice had prominent weight loss and a lean body mass that was approximately $25 \%$ lighter than that of their WT littermates (Figure 1D and Supplemental Figure 1E). Similar to patients with DMD, COUP-TFII OE mice had apparent spinal curvature (kyphosis), indicative of paraspinal muscle weakness (Figure 1E). To further assess whole-body muscle strength and endurance, mice underwent treadmill tests, and the running time and distance to exhaustion were recorded. COUP-TFII-expressing mice ran for a significantly shorter time and distance than did their control counterparts, underscoring a progressive myopathy in the mutants (Figure $1 \mathrm{~F}$ ).

Histologically, muscles in the COUP-TFII OE mice were enriched with small myocytes, and their fiber size distribution showed the typical leftward shift toward smaller fibers (Figure 1, G and H, and Supplemental Figure 1F), implying that the decreased mean cross-sectional area (CSA) may partially account for the muscle atrophy in our transgenic animals. Moreover, fibers from COUP-TFII OE mice harbor additional histopathological signatures of muscular dystrophy such as the appearance of centralized myonuclei, the increment of embryonic myosin heavy chain $\left(\mathrm{eMHC}^{+}\right)$immature fibers, and intramuscular fibrosis (Figure 1, G and I, and Supplemental Figure 1G). During postnatal muscle development, the first 3-4 weeks are the most dynamic period in which $\mathrm{PAX} 7^{+}$cells actively proliferate and continuously incorporate into growing muscles $(26,27)$. Given that ectopic COUP-TFII protein was induced in juvenile SCs on post- 
A

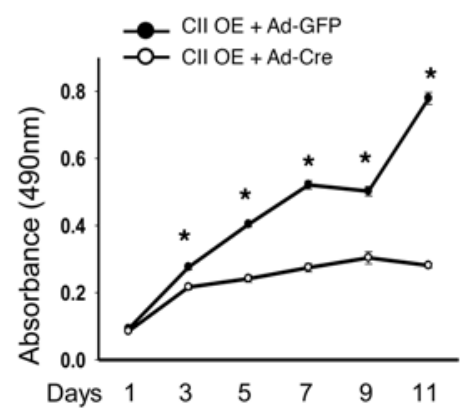

D

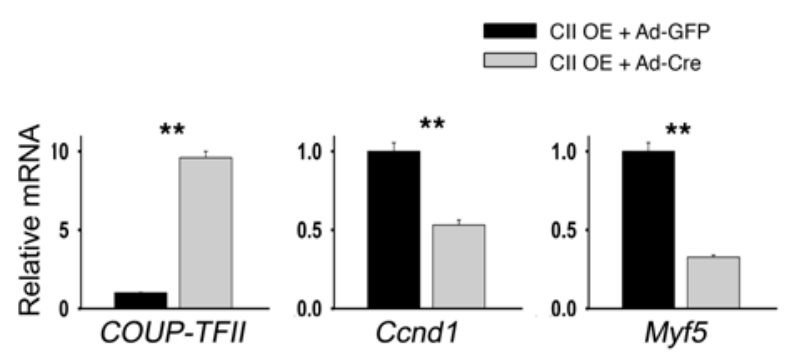

$\mathbf{F}$

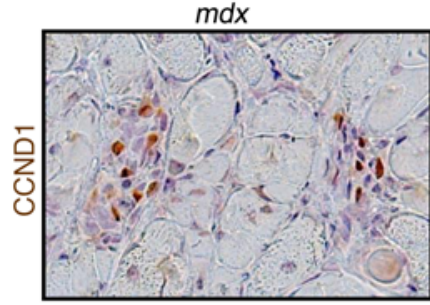

B

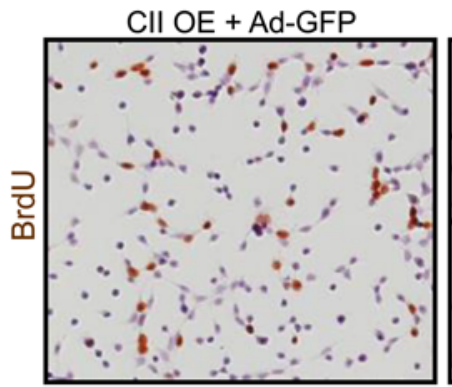

Cll OE + Ad-Cre

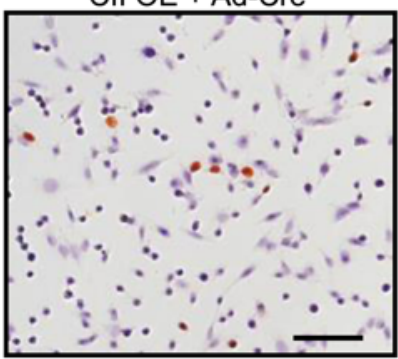

E
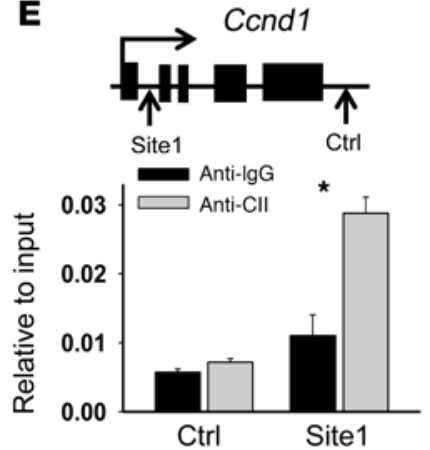

G

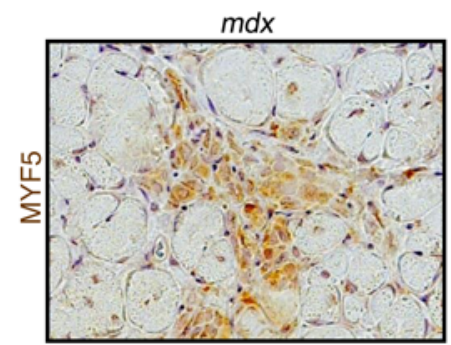

C

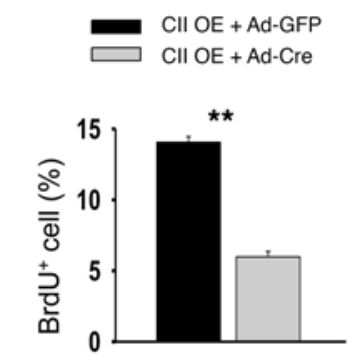

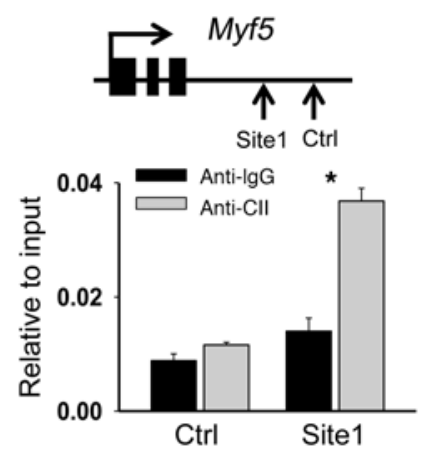

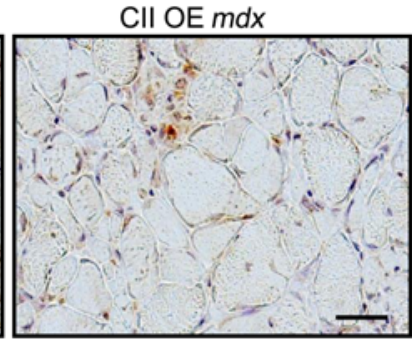

Figure 4. Skeletal muscle progenitors with COUP-TFII dysregulation display proliferative defects. (A) Control and COUP-TFII-expressing myoblasts were cultured for 11 days after virus infection. Data are representative of 3 independent experiments with 3 replicates at each time point. (B and $\mathbf{C}$ ) BrdU immunostaining and quantification of BrdU incorporation in SC cultures 3 days after COUP-TFII induction. (D) qPCR analyses of the expression of Ccnd1 and Myf5 in COUP-TFII OE myoblasts. Results are representative of 3 independent experiments. (E) Primary SC cultures were subjected to ChIP analyses of COUP-TFII occupancy at the Ccnd1 and Myf5 loci, where the control site, lacking a COUP-TFII-binding motif, served as a negative control. Diagram indicates COUP-TFII-binding sites. Results are representative of 3 independent experiments. (F and G) Diaphragm muscles stained with CCND1 and MYF5 Abs. Scale bars: $50 \mu \mathrm{m}$ (B), $25 \mu \mathrm{m}$ (F and $\mathbf{G}$ ). ${ }^{*} P<0.05$ and ${ }^{* *} P<0.01$ by Student's $t$ test. Data represent the mean \pm SEM.

natal day 10 , it is not surprising that a large portion of differentiated myotubes were marked with the Myc-tagged transgene over time (Supplemental Figure $1 \mathrm{H}$ ), raising an interesting possibility that sustained expression of COUP-TFII beyond the stem/progenitor cell stage may also contribute to the DMD-like symptoms observed in our transgenic animals.

Accelerated dystrophic phenotypes in COUP-TFII OE mdx mice. To produce a mouse model that more closely resembled more the conditions seen in patients in whom COUP-TFII was upregulated in conjunction with dystrophin mutation, we bred the COUP-TFII transgene into the murine $m d x$ background. Previous studies have documented that the $m d x$ mouse diaphragm reproduced the pathological changes typical of DMD in humans (6), therefore, we focused on the diaphragm muscles in our analyses. One week after the initial injection of tamoxifen into 4-week-old animals, the Myc-tagged COUP-TFII transgene was mostly found in the SC compartment and was colocalized with PAX7 in COUP-TFII OE and COUP-TFII OE $m d x$ mice (Supplemental Figure 2, A-D). By 4 months of age, muscle hypertrophy and fiber regeneration were evident in the dystrophic diaphragms, whereas forced expression of COUP-TFII impaired tissue recovery, as indicated by obvious muscle atrophy with a few centrally nucleated newborn fibers in the COUP-TFII OE $m d x$ mice (Figure 2, A and B, and Supplemental Figure $3 \mathrm{~A})$. Adult myogenesis recapitulates fetal muscle development by transiently expressing eMHC (3). In line with the de novo fiber synthesis defects, COUP-TFII OE $m d x$ mice had a significantly lower number of differentiating $\mathrm{eMHC}^{+}$cells relative to those detected in control animals (Figure 2, C and D). In $m d x$ mice, SCs actively replicate to provide myonuclear input for newly formed fibers, whereas a decline in progenitor cell proliferation was disclosed in the dystrophic COUP-TFII OE strain through p-histone H3 staining and BrdU labeling (Supplemental Figure 3, 
A
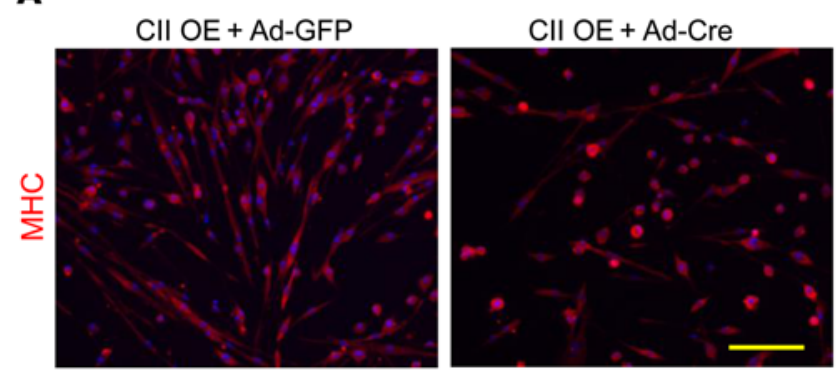

D
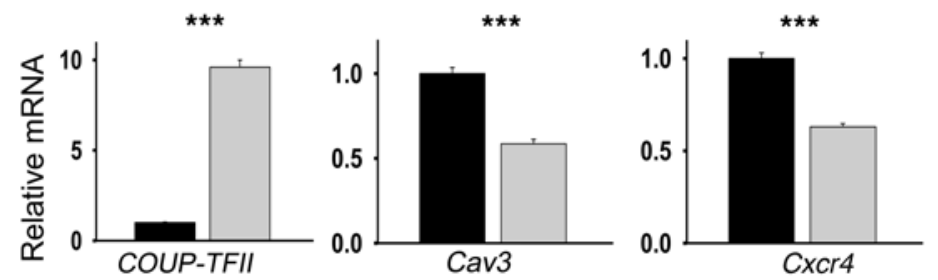

E

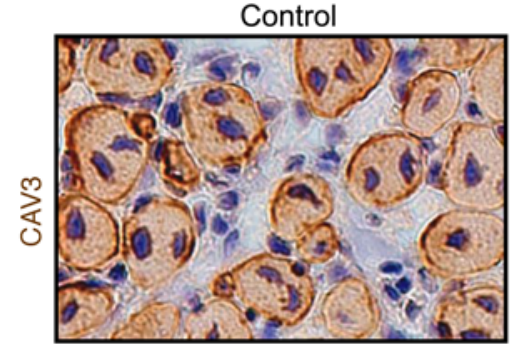

B

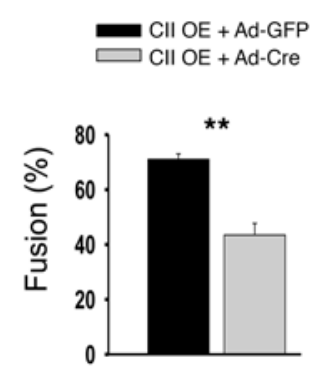

C
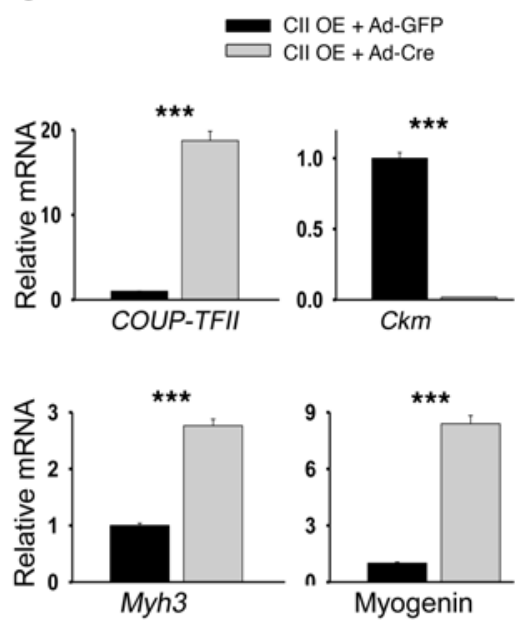

Figure 5. COUP-TFII overexpression impairs myoblast fusion. (A-C) WT and COUP-TFII OE cells were cultured in differentiation medium for 5 days, and myogenic differentiation was assessed by immunostaining against MHC (A), the fusion index (B), and qPCR (C). Results are representative of 3 independent experiments, with 3 replicates for control and COUP-TFII OE cells. The fusion index was calculated as the percentage of myonuclei present in the multinucleated $\mathrm{MHC}^{+}$myotubes. (D) COUP-TFII repressed Cav3 and Cxcr4 transcription in myoblasts. (E) CAV3 staining of regenerating fibers on day 5 after damage. Images are representative of 5 different animals for each genotype. (F) COUP-TFII was recruited to the Cav3 and Cxcr4 enhancer regions. Bar graph showed the enrichment of DNA fragments pulled down by COUP-TFII Ab. Results are representative of 3 independent experiments. Scale bars: $50 \mu \mathrm{m}(\mathbf{A}), 10 \mu \mathrm{m}(\mathbf{E}) .{ }^{*} P<0.05,{ }^{* *} P<0.01$, and ${ }^{* * *} P<0.001$, by Student's $t$ test. Data represent the mean \pm SEM.

B-D). Accordingly, SC homeostasis, enumerated by PAX7 immunoreactivity, dropped by $60 \%$ in COUP-TFII OE $m d x$ animals (Figure 2, E and F).

Muscle regenerative function declines with aging, in which aged SCs fail to activate and proliferate in response to damage signals, thus impeding the reparative process (28). Elevated levels of p16 ${ }^{\mathrm{INK} 4 \mathrm{a}}$ and $\mathrm{p} 19^{\mathrm{ARF}}$ proteins are found in almost all murine tissues with advanced age and are commonly used as biomarkers of in vivo senescence (29). We then assayed $p 16^{I N K 4 a}$ and $p 19^{A R F}$ signals in diaphragm muscles, and comparison of $m d x$ with COUP-TFII OE $m d x$ mice showed no appreciable difference in cellular senescence (Supplemental Figure 4 , A-C). SA- $\beta$-gal is another well-accepted aging-associated marker (30). Consistently, we found that $\beta$-gal density was not altered in $m d x$ animals with or without the COUP-TFII transgene (Supplemental Figure 4D). Similarly, we found no difference in the incidence of apoptosis, as COUP-TFII OE $m d x$ and $m d x$ strains had similar cleaved caspase 3 immunoreactivity (Supplemental Figure 4E). Taken together, we conclude that defective cell expansion probably underlies COUPTFII action in the setting of muscular dystrophy.
The inefficient tissue repair in the compound mutants rendered the tissue unable to keep up with ongoing necrosis, so that muscle wasting occurred. Consistently, lean body mass was reduced by 1.5 fold in $m d x$ mice carrying the COUP-TFII minigene (Figure 2G and Supplemental Figure 4F). Because of muscle hypotrophy, COUP-TFII OE $m d x$ mice had worse dystrophic signs and showed severe kyphosis (Figure $2 \mathrm{H}$ ). Likewise, treadmill and grip tests further demonstrated a profound loss of muscle strength and performance in the compound mutants (Figure 2, I and J). Collectively, we observed exaggerated DMD pathology when COUP-TFII overexpression cooperated with dystrophin mutation, whereby elevated COUP-TFII activity largely abolished the SCmediated reparative response, leading to severe muscle wasting and acceleration of DMD progression.

COUP-TFII negatively regulates regenerative myogenesis. Next, we investigated how the continuous presence of COUP-TFII affects the outcome of transient muscle damage. To do this, 6-week-old control and COUP-TFII OE mice were treated with tamoxifen for 5 consecutive days, and the tibialis anterior (TA) 
A

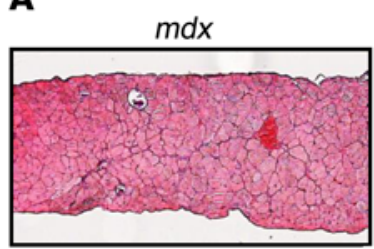

C

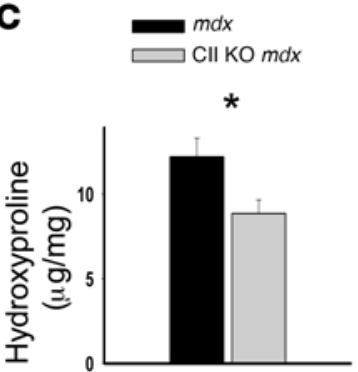

$\mathbf{F}$

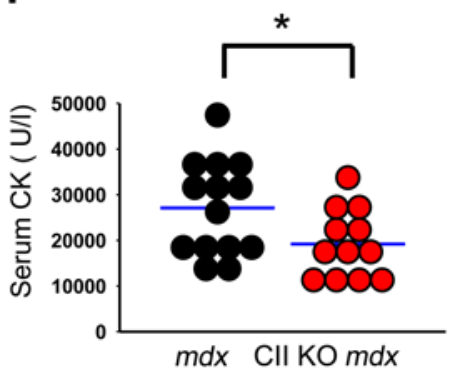

D
$\mathrm{Cll} \mathrm{KO} m d x$
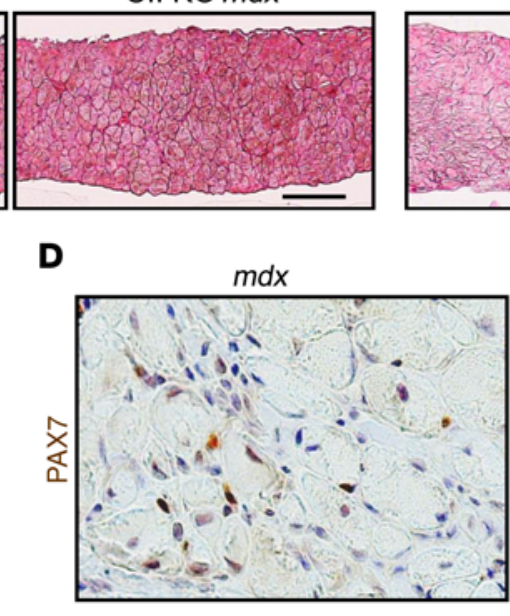

G

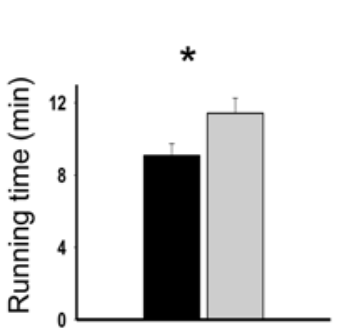

B

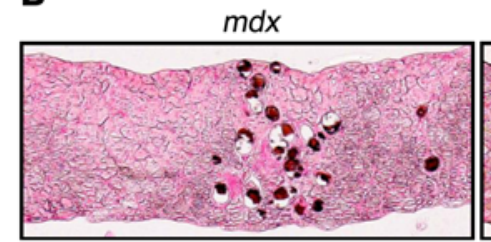

CII KO mdx
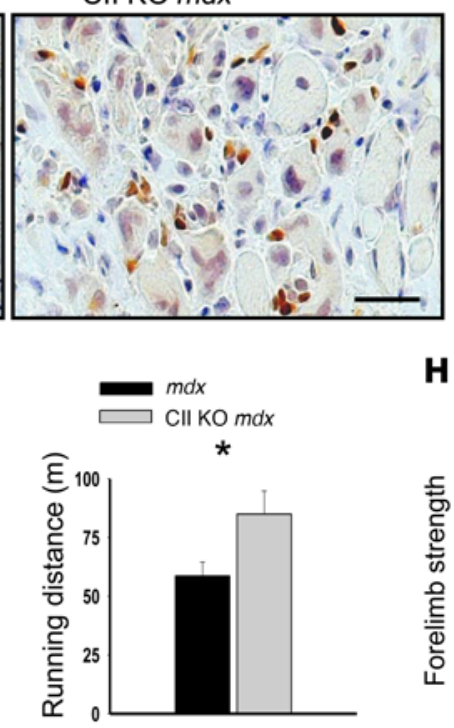

E
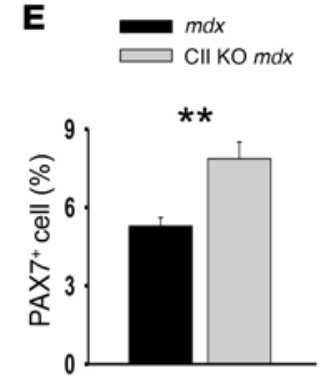

H

$\mathrm{Cll} \mathrm{KO} m d x$

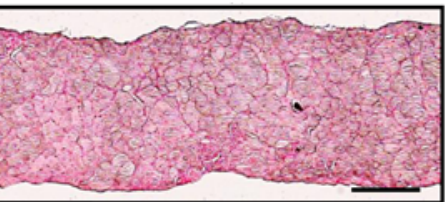

H
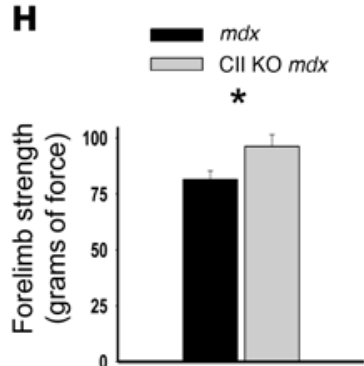

Figure 6. Protection from dystrophic muscle disease in COUP-TFII-KO $\boldsymbol{m d x}$ mice. (A and B) H\&E- and von Kossa-stained 4-month-old diaphragm muscles. Images are representative of 4 different animals for each genotype. (C) Hydroxyproline content ( $\mu$ g hydroxyproline/mg muscle) in diaphragm muscles in $m d x(n=9)$ and COUP-TFII-KO $m d x(n=6)$ mice at 5 to 6 months of age. ( $\mathbf{D}$ and $\mathbf{E})$ PAX7 staining of the diaphragm muscle and the number of PAX7 $7^{+}$nuclei per 100 nuclei in $m d x(n=7)$ and COUP-TFII-KO $m d x(n=6)$ mice at 4 months of age. (F) Quantification of serum CK levels in mdx $(n=14)$ and COUP-TFII-KO $m d x(n=12)$ mice. (G) Ten-month-old $m d x(n=14)$ and COUP-TFII-KO mdx $(n=15)$ animals were subjected to treadmill running tests. (H) Grip test in $m d x(n=10)$ and COUP-TFII KO $m d x(n=12)$ mice. Ten-month-old male mice were used for each genotype cohort. Scale bars: $100 \mu \mathrm{m}$ (A and $\mathbf{B}$ ), $25 \mu \mathrm{m}$ (D). ${ }^{*} P<0.05$ and ${ }^{*} P<0.01$, by Student's $t$ test. Data represent the mean \pm SEM.

muscles were damaged via cardiotoxin (CTX) injection 1 week later. Eight days after injury, necrotic muscles in the control animals were efficiently replaced by newly synthesized fibers (Figure 3A). In striking contrast, TA muscles in COUP-TFII OE mice were filled with small, regenerating myoblasts and abundant mononucleated cells (Figure 3A). By day 12, developing WT myocytes were evenly spaced and uniformly sized, a sign of their maturation (Figure $3 \mathrm{~A}$ ). Conversely, COUP-TFII OE muscles had small fiber diameters and immature development (Figure 3, A and B, and Supplemental Figure 5A). Likewise, muscle repair triggered by barium chloride $\left(\mathrm{BaCl}_{2}\right)$ injury was also compromised with upregulation of COUPTFII (Supplemental Figure 5B).

Muscle reconstitution demands SC activation, proliferation, differentiation, and fusion in an orderly fashion (2). Dysfunction of one or more of these processes could lead to the regenerative failure seen in COUP-TFII-transgenic animals. To explore the mechanistic action of COUP-TFII, we first examined the expression pattern of COUP-TFII after the regenerative insult. As shown in Supplemental Figure 5C, COUP-TFII mRNA levels were high in uninjured muscles but rapidly diminished in the cell-expansion phase, suggesting that COUP-TFII reduction may facilitate myoblast proliferation. In support of this view,
COUP-TFII OE mice displayed a slower rate of cell propagation compared with that seen in WT muscles, as evidenced by BrdU incorporation (Figure 3, C and D), quantitative PCR (qPCR) of cell-cycle-related genes, and p-histone $\mathrm{H} 3$ immunostaining (Supplemental Figure 5, D and E). Acute injury provokes a relatively synchronous regenerative program, in which the changes in cell proliferation in tissues largely reflect the difference within the SC population (2). Correspondingly, COUP-TFII-transgenic mice had a dramatic reduction $(60 \%)$ in the number of $\mathrm{PAX7}^{+}$ cells on day 5 after injury (Figure 3, E and F).

During tissue recovery, mononucleated myoblasts have to fuse with each other or with preexisting fibers to form multinucleated myotubes $(2,3)$. Twelve days after injury, eMHC expression declined in mature $\mathrm{MHC}^{+}$fibers in the control muscles, while fibers in COUP-TFII OE mice maintained strong eMHC signals, reflecting inefficient myoblast fusion and delayed maturation (Figure $3 \mathrm{G}$ ). In addition to SCs, other non-PAX7 ${ }^{+}$progenitors may also give rise to mature myotubes during fiber reconstitution (31, 32). Lineage-tracing experiments using a Myc tag clearly indicated that almost all the centrally nucleated fibers in COUP-TFII OE mice were originated from $\mathrm{Myc}^{+}$precursors (SCs) in which COUPTFII was continuously expressed (Figure 3G), providing evidence 
that sustained COUP-TFII activation indeed impedes lineage progression at the fusion stage. Most strikingly, 2 months after the initial chemical injury, TA muscles in COUP-TFII OE mice were predominantly composed of small, immature $\mathrm{eMHC}^{+}$fibers and widespread fibrotic tissues (Figure $3 \mathrm{H}$ ), emphasizing a permanent destruction of muscle maturation and remodeling under the conditions in which COUP-TFII signaling was high.

COUP-TFII represses the proliferative capacity of muscle stem cells. SCs purified through FACS can proliferate, differentiate, and form multinucleated myotubes. To minimize the potential variations in cell stage and genetic background, we isolated primary SCs from mice carrying a COUP-TFII over- or underexpression allele, which mostly expressed PAX7 protein and readily differentiated into myotubes upon induction (Supplemental Figure $6, \mathrm{~A}$ and B). Next, we asked whether we could reproduce the in vivo proliferation deficits using in vitro cultures. To this end, we infected SCs derived from CAG-S-COUP-TFII mice (23) with adenovirus expressing either GFP (Ad-GFP) or Cre recombinase (AdCre) to induce COUP-TFII protein expression. Eleven days after viral delivery, control myoblasts underwent a 9-fold amplification compared with a 2-fold increase in Ad-Cre-infected COUP-TFII OE cells (Figure 4A). This growth arrest was mainly due to low cell proliferation rates, as the percentage of $\mathrm{BrdU}^{+}$cells was decreased by 2.3 fold after COUP-TFII induction (Figure 4, B and C). Consistently, cell-cycle-associated genes such as cyclins, cyclin-dependent kinases, and their downstream effectors were coordinately downregulated in COUP-TFII-overexpressing cells 3 days after virus infection (Supplemental Figure 6C).

Among those suppressed cell-cycle regulators, Ccnd1, a key player of cell-cycle progression (33), and $M y f 5$ were upregulated in the COUP-TFII-silencing cells (Figure 4D and Supplemental Figure 6D). Myf5 encodes a key myogenic factor that not only delimits the onset of postnatal myogenesis but also supports myoblast expansion during regeneration (34). Similar to the in vitro data, cycling SCs in COUP-TFII-transgenic mice had lower Ccnd1 and Myf5 expression levels than did their control mice (Supplemental Figure 5D). It has been documented that COUP-TFII represses downstream targets via direct binding to the consensus AGGTCA motif with variable spacing (7). Through a ChIP assay, we identified and validated the putative COUP-TFII response elements in the evolutionarily conserved region on the $C c n d 1$ and $M y f 5$ genes, supporting a direct control of these molecules by COUP-TFII (Figure $4 \mathrm{E}$ ). As is the case in an acute injury paradigm (Supplemental Figure 5D), CCND1- and MYF5-immunoreactive cells were more frequently detected in muscles from $m d x$ strains than in muscles from COUP-TFII OE $m d x$ strains (Figure 4, F and G, and Supplemental Figure 6, E and F).

Insulin-like growth factor 1 (IGF-1) has been shown in muscle stem cells to exert pleiotropic functions including cell migration, proliferation, and differentiation (3). As previously reported (35, 36), IGF-1 signals were specifically but transiently detected in activated SCs and small, regenerating myocytes in dystrophic diaphragm muscles (Supplemental Figure 7A). As noted above, COUP-TFII derepression largely blocked dystrophin loss-induced muscle repair (Figure 2 and Supplemental Figure 3), so it was expected that COUP-TFII OE $m d x$ mice would have reduced numbers of IGF- $1^{+}$cells in comparison with numbers in $m d x$ con- trols (Supplemental Figure 7A). However, IGF-1 was not directly controlled by COUP-TFII, because IGF-1 mRNA did not change in COUP-TFII-dysregulated SCs (Supplemental Figure 7, C and D). This conclusion is further supported by the fact that IGF-1 expression was similar on a per-cell basis between control and COUPTFII OE $m d x$ mice (Supplemental Figure 7A). Another secreted growth factor, granulocyte-CSF (G-CSF), has been shown to promote $\mathrm{SC}$ expansion and to maintain a long-term regenerative response in dystrophic animals $(37,38)$. Analysis of G-CSF expression via IHC showed no difference between COUP-TFII OE $m d x$ mice and their control counterparts (Supplemental Figure 7B). Likewise, G-CSF transcript levels did not alter in the COUP-TFII over- or underexpressing cells (Supplemental Figure 7, C and D).

Deficient myoblast fusion in COUP-TFII-expressing cells. During muscle reconstitution, fusion of singly nucleated myoblasts into multinucleated myocytes is indispensable for restoring normal musculature and contractile strength $(39,40)$. A recurrent defect occurring in COUP-TFII-transgenic mice is the loss of myofiber size and strength, implicating an obligate cell-intrinsic function for COUP-TFII in muscle maturation. In accordance with the histological characterizations (Figure 3), qPCR results also indicated a shift in gene expression from mature myotubes ( Ckm and $M y h 2)$ to immature myoblasts ( $M y h 3$ and myogenin) in COUP-TFII OE muscles (Supplemental Figure 8A), supporting the notion of a potential link between defects in myoblast fusion and high levels of COUP-TFII.

To directly assess whether COUP-TFII is a potent factor disrupting myotube formation, we used an SC differentiation assay, in which the fusion process can be recapitulated in vitro. As presented in Figure 5A, myoblasts harboring the COUP-TFII transgene displayed a marked decline in their fusion capacity. In particular, $73 \%$ of cells in the control preparation developed into myotubes containing 2 or more myonuclei, but only $43 \%$ of the COUP-TFII-expressing myoblasts fused to myotubes (Figure 5B). Concordantly, fusion-incompetent COUP-TFII OE cells lacked the induction of the terminal differentiation marker creatine kinase $(\mathrm{Ckm})$ with persistent expression of the early myogenic genes $M y h 3$ and myogenin (Figure 5C).

To define the molecular targets mediating COUP-TFII action, we screened genes critical for myoblast fusion $(39,40)$ and found that the expression of Cav3 and Cxcr 4 was dysregulated in COUPTFII-mutant cells (Figure 5D and Supplemental Figure 8B). Caveolin 3 (CAV3) is a muscle-specific form of the caveolin gene family, and Cav3-deficient cells cannot undergo appropriate myoblast fusion (41, 42). Cxcr 4 encodes a chemokine receptor that is required for myotube formation and muscle regeneration (43). Thus, the transcriptional repression of Cav3 and CXcr 4 by COUPTFII will hamper muscle cell maturation. Significantly, Cxcr 4 and Cav3 were clearly downregulated in regenerating COUP-TFII OE muscles (Supplemental Figure 8A). In WT mice, caveolin 3 was detected at the sarcolemma of newborn fibers, while its sarcolemma-specific expression was either missing or dramatically reduced in our transgenic mice (Figure $5 \mathrm{E}$ ). To determine whether Cav3 and CXcr4 are direct downstream targets of COUP-TFII, we performed ChIP-qPCR analyses and demonstrated the binding of COUP-TFII to the Cav3 and Cxcr4 gene enhancers/promoters (Figure $5 \mathrm{~F}$ ). In luciferase assays, ablation of COUP-TFII pro- 
moted the Cxcr4 enhancer-driven reporter activity, while mutations in the putative COUP-TFII-binding motif partially abolished the induction (Supplemental Figure 8C), corroborating the link between COUP-TFII occupancy and gene regulation. Altogether, our experiments provide compelling evidence that COUP-TFII exerts a critical regenerative impact over the control of Ccnd1, $M y f 5, C a v 3$, and Cxcr4 genes.

Reduction of COUP-TFII activity attenuates the degenerative myopathies in mdx mice. Motivated by the idea that loss of COUPTFII might confer a protective effect in muscular dystrophy, we generated mouse models having a DMD mutation on the COUPTFII $^{-/}$(COUP-TFII-KO) background, in which COUP-TFII mRNA levels were reduced more than $90 \%$ within the SC population (Supplemental Figure 9A). In general, SC-specific COUP-TFIIKO mice did not display overt abnormalities at baseline and had normal muscle size and architecture (Supplemental Figure 9B). Next, we assayed COUP-TFII deletion efficiency in the diaphragm muscle in COUP-TFII-KO $m d x$ mutants and found that COUPTFII protein was largely absent in $\mathrm{PAX} 7^{+}$cells 7 days after tamoxifen administration (Supplemental Figure 9C).

Three months after tamoxifen-mediated recombination, histology of the $m d x$ diaphragm revealed extensive mineralization and muscular fibrosis, while COUP-TFII ablation in dystrophic animals ameliorated DMD pathology including reduced calcium deposits and matrix accumulation (Figure 6, A and B, and Supplemental Figure 10A). As a quantitative measure of collagen density, the mean hydroxyproline content was $25 \%$ lower in COUP-TFII-KO $m d x$ diaphragms than that in control $m d x$ mouse diaphragms (Figure 6C). Excessive matrix accumulation (fibrosis) is a particular hallmark of DMD patients that becomes more severe over time. By 11 months of age, a similar but more prominent reduction in collagen was seen in both diaphragm (Supplemental Figure 10B) and TA (Supplemental Figure 10, C and D) muscles in double-KO animals.

Contrary to the decreased SC density in COUP-TFII OE $m d x$ mutants (Figure 2, E and F), immunostaining for PAX7 uncovered a $40 \%$ increase in PAX7-expressing cells in COUP-TFII-null dystrophic mice (Figure 6, D and E), implying a hastened and longlasting regenerative response in the absence of COUP-TFII. Of note, BW and lean body mass did not differ in 4-month-old dystrophic animals with or without COUP-TFII (Supplemental Figure 11A). Intriguingly, whole-body muscle breakdown, as determined by serum creatine kinase (CK) activity, was significantly lower in COUP-TFII-KO $m d x$ mice as compared with their $m d x$ counterparts (Figure 6F). Sarcolemma integrity, examined by Evans blue dye (EBD) infiltration, confirmed the reduction of muscle damage by COUP-TFII depletion (Supplemental Figure 11, B and C). Histological examination consistently showed overall structural improvements in TA muscles in $m d x$ mice lacking COUP-TFII (Supplemental Figure 12A). Moreover, the number of infiltrating neutrophils (myeloperoxidase [MPO] and Ly-6G) and macrophages (F4/80) was substantially reduced in COUP-TFII-deficient $m d x$ animals (Supplemental Figure 12B), underscoring an attenuation of inflammation, a typical feature of DMD muscles. Those histopathological improvements truly reflected the motor function augmentation, as COUP-TFII-null dystrophic mice were stronger and had better physical activity than did their counter- parts (Figure 6, G and $\mathrm{H}$ ). Together, these parameters support a beneficial effect with COUP-TFII loss, as depletion of COUP-TFII protein mitigates the dystrophic responses and delays the critical hallmarks of DMD, an observation that is consistent with the improved musculature seen in double-KO animals.

\section{Discussion}

SCs are self-renewing muscle stem cells that assure postnatal muscle growth and repair throughout life (27). During neonatal muscle development, $\mathrm{PAX}^{+}$stem/progenitor cells extensively proliferate, differentiate, and fuse with existing fibers, leading to a 4 -fold increase in total muscle mass $(26,44)$. Considering its critical function in myoblast fusion, activation of COUP-TFII in juvenile SCs and its derived myoblasts hampers muscle maturation, with a mean fiber CSA that is decreased by $40 \%$ in advanced age (Figure $1 \mathrm{H}$ and Supplemental Figure $1 \mathrm{~F}$ ). A failure of muscle growth may arise from deficient expansion of individual muscle fibers and/ or a decrease in total fiber numbers (27). Given that high levels of COUP-TFII impair SC expansion (Figure 4), the possible decline of myonuclear accretion during the perinatal growth period probably contributes to the muscle wasting in COUP-TFII OE mice (Figure 1D). Muscle loss is a serious consequence of muscular dystrophy, and COUP-TFII-transgenic animals display additional DMD indices such as central nucleation, fiber size variability, $\mathrm{eMHC}^{+}$ cells, muscular fibrosis, spinal deformity, and exacerbation of muscle dysfunction (Figure 1). As such, we conclude that COUPTFII hyperactivity is indeed a secondary causative factor affecting muscular dystrophy. Reinforcing this notion, forced expression of COUP-TFII further sensitizes the $m d x$ mutants to myopathic changes (Figure 2), emphasizing a synergistic effect when combining COUP-TFII upregulation with dystrophin loss. Together, our findings highlight the contribution of SCs to the pathogenesis of muscular dystrophy and support the perception that, in addition to muscle membrane instability, DMD is also a disease of SC dysfunction, as recently suggested $(45,46)$.

Differentiation of SCs into multinucleated myotubes is a multistep process orchestrated by various myogenic factors and signaling molecules. $M y f 5$ is a muscle-specific transcription factor that directs the myogenic progression of SCs. Previous studies have reported that adult MYF5 depletion leads to progressive myopathy, mild defects in regeneration, and worsened dystrophic pathology in $m d x$ mutants (47), phenotypes that were fully recapitulated by our COUP-TFII-expressing mice (Figure 1-3). However, the dysregulated MYF5 expression can only contribute to, but cannot fully explain, the action of COUP-TFII, as the phenotypes in COUP-TFII OE and COUP-TFII OE $m d x$ mutants are far more severe than those of MYF5-null and $M y f 5^{-1-} m d x$ mice. Therefore, we identified additional targets such as Cav3, Ccnd1, and $\mathrm{Cxcr}$ 4. Caveolin 3 is the genetic basis of autosomal dominant limb-girdle muscular dystrophy (41), and a reduction of CAV3 protein provokes modest fiber degeneration, muscular atrophy, spinal kyphosis, variation in fiber caliber, and slightly increased fibrotic tissue (48-50), symptoms that were phenocopied by COUP-TFII-transgenic animals (Figure 1). Intriguingly, Cav3 is mainly expressed during the differentiation of myogenic cells, and COUP-TFII inhibits Cav3 expression in developing muscles (Figure 5E and Supplemental Figure 8A), pointing to a relevance 
of COUP-TFII derepression in myofiber and muscle dysfunction. Additionally, CXCR4-null mice also display the same limb myogenic defects seen in COUP-TFII OE mice (51). Collectively, the phenotypes seen in $M y f 5, C a v 3$, and Cxcr4 mutants correspond nicely with those of COUP-TFII-transgenic animals, supporting the existence of a regulatory network in which COUP-TFII lies upstream of $M y f 5, C c n d 1, C a v 3$, and Cxcr4 to control SC differentiation and muscle regeneration.

The proliferation of SCs not only produces myogenic progenitors for muscle repair but also maintains the stem cell reservoir. The altered SC homeostasis in COUP-TFII-mutant mice (Figure 2, E and F; Figure 3, E and F; Figure 6, D and E) strongly suggests a requirement of COUP-TFII for replenishing the reserve cell lineage. Consistently, COUP-TFII directly controls the transcripts of Wnt- and Notch-signaling molecules $(9,11)$, key factors dictating the fate of SC progeny (3). Furthermore, $M y f 5$ is a well-known determining factor controlling muscle cell fate specification (2, 3). COUP-TFII suppresses $M y f 5$ expression, hence limiting the degree of myogenic commitment. In agreement with this, COUPTFII-expressing SCs are less prone to entering myogenic paths, as we barely detected $\mathrm{MYF}^{+}$progenitors, $\mathrm{eMHC}^{+}$developing myoblasts, or centralized myonuclei in COUP-TFII OE $m d x$ mice (Figure 2, B-D, Figure 4G, and Supplemental Figure 6F). Thus, it would be interesting to clarify in future studies how COUP-TFII balances stem cell self-renewal and lineage specification.

Although our data favor the interpretation that an autonomous defect within the stem cell compartment leads to muscle dysfunction, several lines of evidence suggest that aberrant COUP-TFII expression on myofibers and myocytes may also contribute to the dystrophy pathology. First, augmented COUP-TFII activity on developing fibers explicitly blocks cell-cell fusion, resulting in muscle atrophy in aged mice as well as regenerative failure in transient injury (Figure 1 and 3). Second, COUP-TFII represses Cav3 expression on myocytes (Figure 5), which in turn elicits mild degenerative responses in COUP-TFII OE animals (Figure 1). A third aspect, the decreased muscle degeneration in COUP-TFIIKO $m d x$ mice, agrees with our observation of an effect of COUPTFII loss in protecting dystrophin-deficient muscles under conditions of stress (Figure 6F and Supplemental Figure 11, B and C). One plausible explanation might invoke the fiber-type switch initiated by COUP-TFII deficit, as dystrophic muscles with a higher degree of slow-twitch fibers are generally more resistant to contractile injury (52). COUP-TFII has been consistently reported to regulate muscle cell metabolism, mitochondrial function, and PGC-1 $\alpha$ expression, key factors controlling muscle fiber types (19, 53). Together, these observations reveal a potential function of COUP-TFII beyond SCs that needs further exploration.

A prerequisite for developing efficacious DMD therapy is the generation of an animal model that faithfully mimics the disease progression in humans. Although they carry the same mutation in the $D M D$ gene, $m d x$ mice have only mild symptoms of the disease $(5,6)$. In the present study, by coupling COUP-TFII hyperactivity with dystrophin loss, we simultaneously targeted 2 major cell types participating in DMD pathology, creating a unique mouse model with exacerbated muscle wasting that more closely approximates that seen in humans. As such, our COUP-TFII OE $m d x$ mice will be a valuable tool in DMD research and preclinical studies. More- over, sustained COUP-TFII expression permanently disrupts the fusion of regenerating myoblasts (Figure $3 \mathrm{H}$ ), which, to our knowledge, has never been reported in other animal models. In this regard, we believe our COUP-TFII-transgenic mice will serve as excellent tools for the investigation of cell-cell fusion in vivo.

In patients with DMD, SC-mediated regeneration initially restores muscle function deterioration. However, such repair is eventually depleted, owing to SC dysfunction. Thus, sustaining the stem cell reservoir and long-term regenerative potential could be an important direction for combating DMD. Here, we show that blunting of COUP-TFII activity stimulates SC lineage, attenuates the dystrophic response, and slows down muscle weakness in $m d x$ mice. COUP-TFII is a member of the nuclear receptor superfamily and contains a ligand-binding pocket that is therapeutically accessible by small, drug-like molecules (54). Hence, it is tempting to speculate that pharmacological blockade of COUP-TFII action using its inhibitors/antagonists might curb muscle wasting and extend patient survival. In summary, we believe our studies provide compelling evidence of novel functions of COUP-TFII in directing SC development and highlight the therapeutic potential of targeting COUP-TFII in DMD or other degenerative muscle disorders.

\section{Methods}

Animals. Mice were bred and housed in a pathogen-free facility. The COUP-TFIILac//+, CAG-S-COUP-TFII, and COUP-TFIIf/fl mice were generated in our laboratory as described previously $(21,23)$. Pax $7^{\text {CreERT2/+ }}$ strains harboring a tamoxifen-inducible CreERT2 fusion under the control of a Pax7 promoter (22) were provided by Charles Keller (Oregon Health \& Science University, Portland, Oregon, USA). The $\mathrm{Pax}^{\mathrm{CreERT2} /+}$ strains were then crossed with mice carrying the $C A G$ $S$-COUP-TFII or COUP-TFIIf/fl allele to generate SC-specific COUPTFII overexpressing (COUP-TFII OE) and underexpressing (COUPTFII-KO) mice, respectively. $m d x$ mice and their control counterpart $C 57 B L / 10 S c S n J$ mice were acquired from The Jackson Laboratory. COUP-TFII OE and COUP-TFII-KO mice were then bred with $m d x$ mutants to produce COUP-TFII OE $m d x$ and COUP-TFII-KO $m d x$ strains. The mice used were at different ages for different experiments, as indicated in the text and figure legends. Mice randomized according to sex were randomly assigned to both the control and test groups, and we ensured that experimental groups were balanced in terms of animal age and sex. In particular, we used both male and female mice in the acute injury paradigm, such as that shown in Supplemental Figure 5C, Supplemental Figure 5D, and Supplemental Figure 8A. Sample size was determined empirically according to previous studies in the assessment of experimental variability. Premature death of animals was the criterion for exclusion. Mice used in the study were on a mixed background; in particular, COUP-TFII OE mice were on a 129SvEv X C57BL/6 background, while COUP-TFII OE $m d x$ and COUP-TFII-KO $m d x$ mice were on a mixed 129SvEv $\times$ C57BL/ $6 \times$ C57BL/10 genetic background. Animals were genotyped by the appropriate PCR-based strategies and were caged together and treated in the same manner. Neither the technician nor the investigator could distinguish between the mice during the experiment or when assessing outcomes. Tamoxifen (Sigma-Aldrich) was dissolved in sunflower seed oil and was injected i.p. into mice at $1 \mathrm{mg}$ per $50 \mathrm{~g}$ of BW for 5 consecutive days.

Histology and IHC. Muscle tissues were dissected, fixed, and processed as paraffin or frozen sections $(11,55)$. For paraffin sec- 
tions, muscles were fixed in $4 \%$ paraformaldehyde overnight and processed for routine paraffin histology. For frozen sections, dissected muscles were briefly fixed in freshly prepared $4 \%$ paraformaldehyde and snap-frozen in liquid nitrogen-cooled isopentane. Tissues were embedded in OCT compound and cut into 10 - $\mu \mathrm{m}$-thick cross sections. Immunostaining was performed with an M.O.M. Kit (Vector Laboratories) following the manufacturer's protocol. Immunofluorescence was conducted using a TSA Kit (Molecular Probes) as described previously (11). $\beta$-Gal staining of the cryostat sections was performed as reported previously (11). $\mathrm{H} \& \mathrm{E}$, trichrome, and von Kossa staining were carried out using standard procedures. For fiber size distribution analyses, H\&Estained images were manually delineated and analyzed using Image J software (NIH) to measure the cross-sectional area of individual muscle fibers ( $>400$ fibers/animal).

The following primary Abs were used: COUP-TFII (1:2,000; PP-H7147; R\&D Systems); PAX7 (1:25; PAX7; DSHB); laminin (1:500; L9393; Sigma-Aldrich); MHC (1:50; MF20; DSHB); eMHC (1:20; F1.652; Developmental Studies Hybridoma Bank [DSHB]); desmin (1:100; M0760; Dako); c-Myc (1:1,000; sc-40; Santa Cruz Biotechnology Inc.); Myc tag (1:100; 2278; Cell Signaling Technology); MYF5 (1:500; sc-302; Santa Cruz Biotechnology Inc.); CCND1 (1:1,000; Rb-9041-PO; NeoMarker); p-histone H3 (1:1,000; 06-570; EMD Millipore); CAV3 (1:2,000; 610420, BD Biosciences); p16 ${ }^{\text {INK4a }}$ (undiluted; 7054713; Roche); p19ARF (1:200; NB200-106; Novus Biologicals); cleaved caspase 3 (1:100; 9661; Cell Signaling Technology); IGF-1 (1:100; 05-172; EMD Millipore); G-CSF (1:100; sc-13102; Santa Cruz Biotechnology Inc.); MPO (1:100; MAB3147; R\&D Systems); Ly-6G (1:500; 550291; BD Pharmingen); F4/80 antigen (1:200; MA516630; Thermo Fisher Scientific); Alexa Fluor 594 donkey anti-mouse IgG (1:500; A-21203; Invitrogen, Thermo Fisher Scientific); and Alexa Fluor 488 goat anti-rabbit IgG (1:500; A-11034; Invitrogen, Thermo Fisher Scientific).

For in vivo BrdU labeling, mice were anesthetized and weighed. A single dose of BrdU (GE Healthcare) reagent was i.p. injected at $1 \mathrm{ml} / 100 \mathrm{~g}$ of BW into control and COUP-TFII OE mice 2 days after damage, and TA muscles were collected 2 hours later. For chronic injury models, diaphragm muscles were collected 20 hours after BrdU administration. Immunostaining for BrdU Ab was performed following the protocol provided by the manufacturer (550803; BD Biosciences).

Senescence-associated $\beta$-gal activity was detected in frozen sections using the Senescence $\beta$-Galactosidase Staining Kit (Cell Signaling Technology). Hydroxyproline content was determined following a protocol provided on the TREAT-NMD website (http://www.treat-nmd.eu/).

SC isolation, culture, differentiation, and staining. SC isolation was performed as previously reported (55), and Thomas Rando of Stanford University (Stanford, California, USA) provided the detailed protocol. Briefly, after sacrifice, hind-limb skeletal muscles were removed, mechanically disaggregated, and dissociated using gentleMACS Dissociator (Miltenyi Biotec). The muscle suspension was then digested with collagenase and dispase. Digested muscles were filtered through a $40-\mu \mathrm{m}$ cell strainer and washed to yield a mononuclear cell suspension. The cells were stained with Vcambiotin (1:75; 553331; BD Biosciences); CD31-FITC (1:75; 553372; BD Biosciences); CD45-FITC (1:75; 553080; BD Biosciences); and Sca1-FITC (1:75; 553335; BD Biosciences). Streptavidin-PE-cy7 (1:75; 557598; BD Biosciences) was used to amplify the Vcam signal. Cells were sorted using a FACSAria II cell sorter (BD Biosciences) at the Cytometry and Cell Sorting Core of Baylor College of Medicine. Isolated SCs were either directly used for RNA extraction or cultured for proliferation and differentiation assays.

SCs isolated from CAG-S-COUP-TFII and COUP-TFII ${ }^{\mathrm{f} / \mathrm{l} / \mathrm{l}}$ mice were cultured in growth medium containing Ham's F10, 20\% FBS, bFGF (5 $\mathrm{ng} / \mathrm{ml}$ ) (R\&D Systems), and 1\% penicillin-streptomycin as previously described (55). Primary SCs were treated with adenovirus expressing GFP or Cre recombinase to activate or ablate the COUP-TFII gene. The virus-infected cells were then transferred to differentiation media (DMEM supplemented with $2 \%$ horse serum and $1 \%$ penicillin-streptomycin) to induce myogenic development. Differentiated SCs were stained with MHC Ab to visualize multinucleated myotubes. The fusion index was calculated as the percentage of nuclei within myotubes containing at least 2 nuclei as a ratio of total nuclei in the field.

For immunostaining of myotubes, cells were grown on culture slides (BD Biosciences) in induction medium for 5 days. Cells were then fixed with $4 \%$ paraformaldehyde, washed, and permeabilized with $0.3 \%$ Triton X-100 for 10 minutes. Cells were blocked with $10 \%$ goat serum for 30 minutes, followed by incubation with MHC Ab (1:50; MF20; DSHB) for 2 hours. Cells were washed in PBS and incubated with the secondary Ab Alexa Fluor 594 donkey anti-mouse IgG for 1 hour. After washing, culture slides were mounted with Vector Laboratories Mounting Medium. BrdU staining was conducted using the BrdU In-Situ Detection Kit (BD Biosciences). BrdU incorporation was determined as the percentage of $\mathrm{BrdU}^{+}$cells to total nuclei in the field.

Muscle damage, serum CK, and EBD measurements. CTX from Naja mossambica and $\mathrm{BaCl}_{2}$ (Sigma-Aldrich) was dissolved in sterile saline to a final concentration of $10 \mu \mathrm{M}$ and $1.2 \%$, respectively. CTX and $\mathrm{BaCl}_{2}$ were aliquoted and stored at $-20^{\circ} \mathrm{C}$. Before injury, mice were anesthetized by isoflurane inhalation or i.p. injection of 2.5\% Avertin (Sigma Aldrich). Mouse legs were shaved and cleaned with alcohol. TA muscle was injected with $100 \mu \mathrm{CTX}$ or $\mathrm{BaCl}_{2}$ using an insulin syringe. After injection, animals were kept on a heating pad until recovery. The damaged TA muscles were collected at the indicated times (Figure $3 \mathrm{~A}$ and Supplemental Figure 5B ) and stained with $\mathrm{H} \& \mathrm{E}$ using routine procedures.

Serum CK activity was analyzed as an overall measure of wholebody muscle breakdown. Four-month-old animals were anesthetized, and blood was obtained from the periorbital vascular plexus. Serum CK levels were measured by the Comparative Pathology Laboratory at Baylor College Medicine.

To evaluate muscle fiber integrity, $1 \%$ EBD dissolved in sterile saline was administered by i.p. injection at a dose of $1 \%$ of BW. Twenty hours later, muscle was dissected, processed, and cut for histological analyses. Muscle sections were stained with laminin to visualize muscle fiber, and EBD was detected as red autofluorescence.

$R N A$ extraction and quantitative real time $P C R$ ( $R T-q P C R)$. Total RNA was isolated from SCs and TA muscles using TRIzol reagent (Invitrogen, Thermo Fisher Scientific). RNA was then digested with DNase and reverse transcribed with random primers using SuperScript II reverse transcriptase (Invitrogen, Thermo Fisher Scientific). Expression of genes of interest was assessed using standard qPCR approaches with either inventoried TaqMan primer/probe mix or SYBR Green-based realtime PCR. Reactions were run in triplicate, and automatically detected threshold cycle $(\mathrm{Ct})$ values were compared among samples. Levels of gene expression were normalized to the level of 18s rRNA, Gapdh, or cyclophilin A housekeeping genes. TaqMan probes were all from Invitro- 
gen (Thermo Fisher Scientific): COUP-TFII (Mm00772789_m1); Ccnd1 (Mm00432359_m1); Myf5 (Mm00435125_m1); Ckm (Mm01321487_ m1); myogenin (Mm00446194_m1); Myh3 (Mm01332463_m1); Cav3 (Mm00725536_s1); Cxcr4 (Mm01996749_s1); Mki67 (Mm01278617 m1); Ccne1 (Mm01266311_m1); E2f1 (Mm00432939_m1); Pax7 (Mm01354484_m1); 18s rRNA (4319413E); Igf1 (Mm00439560_m1); GCSF (Mm00438334_m1); Ccnd2 (Mm00438070_m1); Ccnd3 (Mm01612362_m1); Cdk4 (Mm00726334_s1); Cdk6 (Mm01311342_ m1); Ccna1 (Mm00432337_m1); Ccna2 (Mm00438063_m1); Cdk2 (Mm00443947_m1); Ccnb1 (Mm03053893_g); Cdc25a (Mm00483162_ $\mathrm{m1}) ; \quad C d c 25 b \quad$ (Mm00499136_m1); $\quad C d c 25 c \quad$ (Mm00486872_m1); Cdc6 (Mm03048221_m1); Mcm3 (Mm00801867_m1); and Myh2 (Mm01332564_m1). The following SYBR Green primers were used: cyclophilin A (forward) 5'-TTGCCATTCCTGGACCCAAA-3', (reverse) 5'-ATGGCACTGGTGGCAAGTCC-3'; Gapdh (forward) 5'-GGCAAAGTGGAGATTGTTGC-3', (reverse) 5'-AATTTGCCGTGAGTGGAGTC-3'; Lmnb1 (forward) 5'-CTTCGAGAGTATGAGGCGGC-3', (reverse) 5'-TAACTGCTTTTTGGCGGCAG-3'; and Cav3 (forward) 5'-CACAAGGCTCTGATCGCCTC-3', (reverse) 5'-TCCGTGTGCTCTTCGGTCA-3'.

ChIP. ChIP assays were performed in cultured SCs following the protocol provided by EMD Millipore with specific anti-COUP-TFII (R\&D Systems) and anti-mouse IgG Ab (EMD Millipore). Bound fraction and input DNA were subjected to qPCR using the following primers: $C c n d 1$ control primer (forward) 5'-CCAGAAATACAAAAAGCCAACC-3', (reverse) 5'-CCAGGCAGGACCCTTGAG-3'; Cond1 ChIP primer (forward) 5'-GGTGCCTAAAAACGACCTGA-3'，(reverse) 5'-CCCTTGTAGAGGCAAACAGC-3'; Myf5 control primer (forward) 5'-TGGAGAAGTTGGTTTCCACTC-3', (reverse) 5'-TCCTCAGTTTCCCACTAGCC-3'; Myf5 ChIP primer (forward) 5'-TACCAGGCAGTTCATCACCA-3', (reverse) 5'-ACCAATGTCTCCCCTTCCTT-3'; Cav3 control primer (forward) 5'-CCTGGATTTGAGTCCCAATG-3', (reverse) 5'-CATCTCGAGGTGGCTTGTTA-3'; Cav3 ChIP primer (forward) 5'-GAGAACCATGTCCCCATCCT-3', (reverse) 5'-CACTTGGCTGATGGGCTACT-3'; Cxcr4 control primer (forward) 5'-CAGCAGAGGTTAGCCAACAG-3', (reverse) 5'-GCAAAAGGGTTTGGGAAAGT-3'; and Cxcr4 ChIP primer (forward) 5'-TGAAAACATTGCGTTTGAGG-3', (reverse) 5'-CTTGGACGGCTTCCAGATAG-3'.

$X$-ray, body composition, treadmill, and grip tests. The treadmill test was performed using the Exer-6M (Columbus Instruments) at a 10-degree incline (Figure $1 \mathrm{~F}$ and Figure 2I) or following the protocol suggested by the TREAT-NMD network (Figure 6G). Exhaustion was defined by the inability of the animal to remain on the treadmill despite electrical prodding. To assess mouse forelimb strength, we used a Grip Strength Meter (Columbus Instruments) and carried out grip tests as recommended by the TREAT-NMD protocol (http:// www.treat-nmd.eu/research/preclinical/dmd-sops/). The mice were allowed to grip the horizontal bar and were pulled horizontally by the tail until the bar was released, then the peak force was recorded for each mouse. Body composition assessment, $\mathrm{x}$-ray imaging, and treadmill and grip tests were performed by the Mouse Phenotyping Core at Baylor College of Medicine.

Transfection and luciferase assays. CXCR4 WT enhancer and the mutant form were synthesized by Integrated DNA Technologies and cloned into a pLuc-MCS vector (Agilent Technologies). The predicted COUP-TFII-binding motif was mutated from TAACCTCTAACCT to GTCCTACAATTAG. Myoblasts carrying the COUP-TFII/f/fl allele were first infected with Ad-GFP and Ad-Cre viruses to ablate COUP-TFII protein. WT and mutant CXCR4 luciferase reporters were then transfected into virus-treated SCs using Lipofectamine 2000 (Invitrogen, Thermo Fisher Scientific). Luciferase activity was measured 48 hours later according to the manufacturer's instructions (Promega).

Statistics. All data are shown as the mean \pm SEM of at least 3 biological replicates. Statistical significance was calculated using a 2-tailed Student's $t$ test, assuming normal distribution and equal variance among different samples. Statistics significance is signified in the figure legends as ${ }^{*} P<0.05,{ }^{* *} P<0.01$, and ${ }^{* *} P<0.001$.

Study approval. All animal experimental procedures were reviewed and approved by the IACUC of Baylor College of Medicine, and all experiments were performed in compliance with the institutional guidelines of Baylor College of Medicine.

\section{Author contributions}

$\mathrm{XX}, \mathrm{SYT}$, and MJT conceptualized and designed the study. XX performed and analyzed the experiments. XX, SYT, and MJT wrote, discussed, and edited the manuscript.

\section{Acknowledgments}

We are grateful to Charles Keller (Oregon Health \& Science University) and Thomas Cooper and Ravi Singh (Baylor College of Medicine) for providing $\operatorname{Pax} 7^{\mathrm{CreERT} /{ }^{+}}$mice and for their constructive suggestions. We thank Thomas Rando (Stanford University) for the SC isolation protocols. We appreciate the technical support of Pei $\mathrm{Li}$ and Guannu $\mathrm{Xu}$, and Jodie R. Hebert for her help in editing this manuscript. The Genetically Engineered Mouse Core, Mouse Phenotyping Core, the Cytometry and Cell Sorting Core, and the Comparative Pathology Laboratory at Baylor College of Medicine provided facilities and assistance (P30 DK079638 and P30 CA125123). This work was supported by grants from the NIH (DK59820 and HL114539, to SYT and MJT, and DK45641, to MJT).

Address correspondence to: Sophia Y. Tsai or Ming-Jer Tsai, Department of Molecular and Cellular Biology, Baylor College of Medicine, One Baylor Plaza, Houston, Texas 77030, USA. Phone: 713.798.6251; E-mail: stsai@bcm.tmc.edu (S.Y. Tsai). Phone: 713.798.6253; E-mail: mtsai@bcm.tmc.edu (M.J. Tsai).
1. Scharner J, Zammit PS. The muscle satellite cell at 50: the formative years. Skelet Muscle. 2011;1(1):28.

2. Wang YX, Rudnicki MA. Satellite cells, the engines of muscle repair. Nat Rev Mol Cell Biol. 2012;13(2):127-133.

3. Yin H, Price F, Rudnicki MA. Satellite cells and the muscle stem cell niche. Physiol Rev. 2013;93(1):23-67.
4. Bushby K, et al. Diagnosis and management of Duchenne muscular dystrophy, part 1: diagnosis, and pharmacological and psychosocial management. Lancet Neurol. 2010;9(1):77-93.

5. Hoffman EP, Brown RH, Kunkel LM. Dystrophin: the protein product of the Duchenne muscular dystrophy locus. Cell. 1987;51(6):919-928.

6. Stedman HH, et al. The mdx mouse diaphragm reproduces the degenerative changes of Duchenne muscular dystrophy. Nature. 1991;352(6335):536-539.

7. Tsai SY, Tsai MJ. Chick ovalbumin upstream promoter-transcription factors (COUP-TFs): coming of age. Endocr Rev. 1997;18(2):229-240.

8. Xie X, Tang K, Yu CT, Tsai SY, Tsai MJ. Regulatory potential of COUP-TFs in development: stem/progenitor cells. Semin Cell Dev Biol. 2013;24(10-12):687-693. 
9. Lin FJ, Qin J, Tang K, Tsai SY, Tsai MJ. Coup d'Etat: an orphan takes control. Endocr Rev. 2011;32(3):404-421.

10. Pereira FA, Qiu Y, Zhou G, Tsai MJ, Tsai SY. The orphan nuclear receptor COUP-TFII is required for angiogenesis and heart development. Genes Dev. 1999;13(8):1037-1049.

11. Xie X, Qin J, Lin SH, Tsai SY, Tsai MJ. Nuclear receptor chicken ovalbumin upstream promoter-transcription factor II (COUP-TFII) modulates mesenchymal cell commitment and differentiation. Proc Natl Acad Sci U S A. 2011;108(36):14843-14848.

12. Muscat GE, Rea S, Downes M. Identification of a regulatory function for an orphan receptor in muscle: COUP-TF II affects the expression of the myoD gene family during myogenesis. Nucleic Acids Res. 1995;23(8):1311-1318.

13. Bailey P, Sartorelli V, Hamamori Y, Muscat GE. The orphan nuclear receptor, COUP-TF II, inhibits myogenesis by post-transcriptional regulation of MyoD function: COUP-TF II directly interacts with p300 and myoD. Nucleic Acids Res. 1998;26(23):5501-5510.

14. Bakay M, et al. Nuclear envelope dystrophies show a transcriptional fingerprint suggesting disruption of Rb-MyoD pathways in muscle regeneration. Brain. 2006;129(Pt 4):996-1013.

15. Chen YW, Zhao P, Borup R, Hoffman EP. Expression profiling in the muscular dystrophies: identification of novel aspects of molecular pathophysiology. J Cell Biol. 2000;151(6):1321-1336.

16. Balagopal P, et al. Oxandrolone enhances skeletal muscle myosin synthesis and alters global gene expression profile in Duchenne muscular dystrophy. Am J Physiol Endocrinol Metab. 2006;290(3):E530-E539.

17. de Rezende Pinto WB, de Souza PV, Oliveira AS. Normal muscle structure, growth, development, and regeneration. Curr Rev Musculoskelet Med. 2015;8(2):176-181.

18. Qin J, et al. COUP-TFII inhibits TGF- $\beta$-induced growth barrier to promote prostate tumorigenesis. Nature. 2013;493(7431):236-240.

19. Wu SP, et al. Increased COUP-TFII expression in adult hearts induces mitochondrial dysfunction resulting in heart failure. Nat Commun 2015;6:8245.

20. You LR, et al. Mouse lacking COUP-TFII as an animal model of Bochdalek-type congenital diaphragmatic hernia. Proc Natl Acad Sci U S A. 2005;102(45):16351-16356.

21. Takamoto N, et al. COUP-TFII is essential for radial and anteroposterior patterning of the stomach. Development. 2005;132(9):2179-2189.

22. Nishijo K, et al. Biomarker system for studying muscle, stem cells, and cancer in vivo. FASEB J. 2009;23(8):2681-2690.

23. Wu SP, Lee DK, Demayo FJ, Tsai SY, Tsai MJ. Generation of ES cells for conditional expression of nuclear receptors and coregulators in vivo. Mol Endocrinol. 2010;24(6):1297-1304.

24. Blake DJ, Weir A, Newey SE, Davies KE. Function and genetics of dystrophin and dystrophin-related proteins in muscle. Physiol Rev. 2002;82(2):291-329.

25. Lou JS, Weiss MD, Carter GT. Assessment and management of fatigue in neuromuscular disease. Am J Hosp Palliat Care. 2010;27(2):145-157.

26. White RB, Biérinx AS, Gnocchi VF, Zammit PS. Dynamics of muscle fibre growth during postnatal mouse development. BMC Dev Biol. 2010;10:21.

27. Tajbakhsh S. Skeletal muscle stem cells in developmental versus regenerative myogenesis. JIntern Med. 2009;266(4):372-389.

28. Sousa-Victor P, et al. Geriatric muscle stem cells switch reversible quiescence into senescence. Nature. 2014;506(7488):316-321.

29. Krishnamurthy J, et al. Ink4a/Arf expression is a biomarker of aging. JClin Invest. 2004;114(9):1299-1307.

30. Sousa-Victor P, García-Prat L, Serrano AL, Perdiguero E, Muñoz-Cánoves P. Muscle stem cell aging: regulation and rejuvenation. Trends Endocrinol Metab. 2015;26(6):287-296.

31. Dellavalle A, et al. Pericytes of human skeletal muscle are myogenic precursors distinct from satellite cells. Nat Cell Biol. 2007;9(3):255-267.

32. Sampaolesi M, et al. Mesoangioblast stem cells ameliorate muscle function in dystrophic dogs. Nature. 2006;444(7119):574-579.

33. Serrano AL, Baeza-Raja B, Perdiguero E, Jardí M, Muñoz-Cánoves P. Interleukin-6 is an essential regulator of satellite cell-mediated skeletal muscle hypertrophy. Cell Metab. 2008;7(1):33-44.

34. Ustanina S, Carvajal J, Rigby P, Braun T. The myogenic factor Myf5 supports efficient skeletal muscle regeneration by enabling transient myoblast amplification. Stem Cells. 2007;25(8):2006-2016.

35. Jennische E, Skottner A, Hansson HA. Satellite cells express the trophic factor IGF-I in regenerating skeletal muscle. Acta Physiol Scand. 1987;129(1):9-15.

36. Jennische E, Hansson HA. Regenerating skeletal muscle cells express insulin-like growth factor I. Acta Physiol Scand. 1987;130(2):327-332.

37. Hayashiji N, et al. G-CSF supports long-term muscle regeneration in mouse models of muscular dystrophy. Nat Commun. 2015;6:6745.

38. Hara M, et al. G-CSF influences mouse skeletal muscle development and regeneration by stimulating myoblast proliferation. JExp Med. 2011;208(4):715-727.

39. Hindi SM, Tajrishi MM, Kumar A. Signaling mechanisms in mammalian myoblast fusion. Sci Signal. 2013;6(272):re2.

40. Abmayr SM, Pavlath GK. Myoblast fusion: lessons from flies and mice. Development.
2012;139(4):641-656

41. Gazzerro E, Sotgia F, Bruno C, Lisanti MP, Minetti C. Caveolinopathies: from the biology of caveolin-3 to human diseases. Eur J Hum Genet. 2010;18(2):137-145

42. Galbiati F, Volonte D, Engelman JA, Scherer PE, Lisanti MP. Targeted down-regulation of caveolin-3 is sufficient to inhibit myotube formation in differentiating $\mathrm{C} 2 \mathrm{C} 12$ myoblasts. Transient activation of $\mathrm{p} 38$ mitogen-activated protein kinase is required for induction of caveolin-3 expression and subsequent myotube formation. J Biol Chem. 1999;274(42):30315-30321.

43. Griffin CA, Apponi LH, Long KK, Pavlath GK. Chemokine expression and control of muscle cell migration during myogenesis. J Cell Sci. 2010;123(Pt 18):3052-3060.

44. Gokhin DS, Ward SR, Bremner SN, Lieber RL. Quantitative analysis of neonatal skeletal muscle functional improvement in the mouse. J Exp Biol. 2008;211(Pt 6):837-843.

45. Sacco A, et al. Short telomeres and stem cell exhaustion model Duchenne muscular dystrophy in mdx/mTR mice. Cell. 2010;143(7):1059-1071.

46. Dumont NA, et al. Dystrophin expression in muscle stem cells regulates their polarity and asymmetric division. Nat Med. 2015;21(12):1455-1463.

47. Gayraud-Morel B, Chrétien F, Flamant P, Gomès D, Zammit PS, Tajbakhsh S. A role for the myogenic determination gene Myf5 in adult regenerative myogenesis. Dev Biol. 2007;312(1):13-28.

48. Ohsawa Y, et al. Muscular atrophy of caveolin3-deficient mice is rescued by myostatin inhibition. J Clin Invest. 2006;116(11):2924-2934.

49. Hagiwara Y, et al. Caveolin-3 deficiency cause muscle degeneration in mice. Hum Mol Genet. 2000;9(20):3047-3054.

50. Sunada Y, et al. Transgenic mice expressing mutant caveolin-3 show severe myopathy associated with increased nNOS activity. Hum Mol Genet. 2001;10(3):173-178.

51. Odemis V, et al. Mice deficient in the chemokine receptor CXCR4 exhibit impaired limb innervation and myogenesis. Mol Cell Neurosci. 2005;30(4):494-505.

52. Consolino CM, Brooks SV. Susceptibility to sarcomere injury induced by single stretches of maximally activated muscles of $\mathrm{mdx}$ mice. J Appl Physiol. 2004;96(2):633-638.

53. Myers SA, Wang SC, Muscat GE. The chicken ovalbumin upstream promoter-transcription factors modulate genes and pathways involved in skeletal muscle cell metabolism. J Biol Chem. 2006;281(34):24149-24160.

54. Kruse SW, et al. Identification of COUP-TFII orphan nuclear receptor as a retinoic acid-activated receptor. PLoS Biol. 2008;6(9):e227.

55. Cheung TH, et al. Maintenance of muscle stemcell quiescence by microRNA-489. Nature. 2012;482(7386):524-528. 